\title{
Southern ultraluminous infrared galaxies: An optical and infrared database ${ }^{\star}$
}

\author{
P.-A. Duc ${ }^{1,2}$, I.F. Mirabel ${ }^{2}$, and J. Maza ${ }^{3, \star \star}$ \\ 1 ESO, Karl-Schwarzschild-Str. 2, D-85748 Garching bei München, Germany \\ 2 Service d'Astrophysique, CEA/DSM/DAPNIA, C.E. Saclay, 91191 Gif-sur-Yvette Cedex, France \\ 3 Depto. Astronomia, Univ. de Chile, Casilla 36-D, Santiago, Chile
}

Received October 18; accepted December 16, 1996

\begin{abstract}
We have carried out an optical and infrared study of 24 ultraluminous infrared galaxies in the southern hemisphere (SULIRGs). This flux limited, complete sample, has been extracted from the redshift survey of IRAS galaxies compiled by Strauss et al. (1992). It includes systems with a $60 \mu \mathrm{m}$ IRAS flux greater than 3 Jy and a far-infrared luminosity greater than $6.510^{11} L_{\odot}\left(H_{0}=75 \mathrm{~km} \mathrm{~s}^{-1} \mathrm{Mpc}^{-1}\right)$. With the ESO New Technology Telescope, we have obtained high resolution optical images in the $R$ band of all SULIRGs, and with the ESO/MPI 2.2-meter telescope, near infrared $J$, $H$ and $K$ images for most of them. Low and high resolution spectra have been taken with the 4-meter telescope of the Cerro Tololo Inter-American Observatory. The nature and morphology of all objects are consistent with the wide-spread idea that most, if not all, galaxies with an extreme far-infrared luminosity are close interacting or merging systems. The galaxies that appear as isolated and devoid of tidal features exhibit several isophotal distortions in their main body. However three cases are more ambiguous. They are indeed interacting systems, but their companions are at a distance greater than $40 \mathrm{kpc}$. We find in the SULIRG sample a significant proportion of objects having AGN like spectra: $55 \pm 6 \%$, including one Seyfert 1 galaxy.
\end{abstract}

Key words: galaxies: active - galaxies: interactions infrared: galaxies

Send offprint requests to: pduc@eso.org

* Partly based on observations collected at the European Southern Observatory, La Silla, Chile.

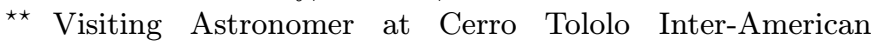
Observatory, operated by AURA Inc., under contract with the National Science Foundation.

\section{Introduction}

Since the availability of the IRAS database, several surveys have been devoted to the search of ultraluminous infrared galaxies (ULIRG), a class of objects with an infrared luminosity above $10^{12} L_{\odot}$, comparable to the bolometric luminosity of optically selected quasars. Some ULIRG samples have directly been selected from IRAS flux catalogs, using distance-independent criteria, such as optical to infrared flux ratios (Clements et al. 1996b) or infrared colors (Lawrence et al. 1986; Kim 1995), making them good ULIRG candidates. Confirmation of their high luminosity was then obtained from spectroscopic followups. Others have been extracted from complete, flux limited, IRAS galaxies redshift surveys. The two more important ones so far published are the IRAS Bright Galaxy Sample (BGS, Soifer et al. 1986) for galaxies with a declination above $30^{\circ}$ and a $60 \mu \mathrm{m}$ flux lower limit of $5.24 \mathrm{Jy}$, extended to the whole sky in the Extended Bright Galaxy Sample (Sanders et al. 1996), and the deeper 1.9 Jy redshift survey by Strauss et al. (1992), which covers the entire sky. In the Bright Galaxy Sample, Sanders et al. (1988a) have found 10 ULIRGs, with an infrared luminosity greater than $10^{12} L_{\odot}$. These galaxies have afterwards been extensively studied in various wavelength ranges (e.g. Carico et al. 1988; Majewski et al. 1993). We present here a sample of ULIRGs, which was extracted from the list of Strauss et al. (1992), restricted to the southern hemisphere, and complete for $60 \mu \mathrm{m}$ fluxes greater than $3 \mathrm{Jy}$. Northern ULIRGs of the $1.9 \mathrm{Jy}$ survey have recently been presented by Murphy et al. (1996). The 24 Southern Ultraluminous Infrared Galaxies (hereafter referred as SULIRG) in the sample have been studied by means of optical and infrared imaging and spectroscopy.

Despite the high number of papers devoted to Ultraluminous Infrared Galaxies (see the recent reviews by Sanders \& Mirabel 1996 and Moorwood 1996), many questions about their nature remain unanswered. In particular the power source of their enhanced 
infrared luminosity - starburst, AGN, combination of both

- is unknown. Their morphological class is even matter of debate. Although it is widely admitted that most ULIRGs are interacting or merging systems, the actual proportion varies greatly according to the the authors (Sanders et al. 1988a; Melnick \& Mirabel 1990; Leech et al. 1994). To that respect, the choice of the ULIRG parent catalogue is of prime importance, since it may introduce biases in statistical studies. Extracting an ULIRG sample from a list of faint IRAS sources, distributed over a limited portion of the sky, Leech et al. (1994) have selected distant objects for which a morphological classification is dubious. Using infrared warm objects, Sanders et al. (1988b) have preferentially chosen active galaxies. To that respect the BGS and 1.9 Jy redshift surveys appear to be more likely to provide with less biased samples of ULIRGs.

Section 2 describes our sample, and Sect. 3, the observations and the data reduction. Some general results dealing with the morphology and the spectral classification of the SULIRGs are presented in Sect. 4. Notes on individual objects are given in Sect. 5 .

\section{The sample of southern ultraluminous galaxies}

The redshift survey compiled by Strauss et al. (1992), from which we have extracted our sample of ultraluminous infrared galaxies, includes objects from the IRAS Point Source Catalog with a $60 \mu \mathrm{m}$ flux greater than $1.936 \mathrm{Jy}$. A flux color constraint was also applied by these authors to exclude stars and galactic sources $\left(f_{60}^{2}>f_{12} f_{25}\right)$. From the 2658 galaxies of the original sample, we have selected the most luminous objects in the far infrared according to the following criteria:

1. Declination, $\delta<0^{\circ}$.

2. Absolute Galactic Latitude, $|b|>15^{\circ}$.

3. $60 \mu \mathrm{m}$ Flux, $f_{60}>3$ Jy.

4. Far Infrared Luminosity, $L_{\mathrm{FIR}}>6.510^{11} L_{\odot}$.

The choice of the southern hemisphere was motivated by the desire to cover a region of the sky not present in the ULIRG sample of Sanders et al. (1988a). The second constraint excludes galaxies contaminated by the galactic plane.

$L_{\text {FIR }}$ was calculated using the formula:

$L_{\mathrm{FIR}}\left(L_{\odot}\right)=5.610^{5} D(\mathrm{Mpc})^{2}\left(2.58 f_{60}+f_{100}\right)$,

which is an approximation of the $\lambda 40 \mu \mathrm{m}-\lambda 120 \mu \mathrm{m}$ luminosity (Lonsdale et al. 1985), and assuming $H_{0}=$ $75 \mathrm{~km} \mathrm{~s}^{-1}{ }^{\prime} \mathrm{Mpc}^{-1}$. A value of $L_{\mathrm{FIR}}=6.510^{11} L_{\odot}$ is comparable to the canonical limit $L_{\mathrm{IR}}=10^{12} L_{\odot}$, originally used in ULIRG studies, where $L_{\mathrm{IR}}$ is an approximation of the $\lambda 8 \mu \mathrm{m}-\lambda 1000 \mu \mathrm{m}$ luminosity, given by Perault (1989), as:

$$
\begin{aligned}
L_{\mathrm{IR}}\left(L_{\odot}\right)= & 5.610^{5} D(\mathrm{Mpc})^{2} \\
& \left(13.56 f_{12}+5.26 f_{25}+2.54 f_{60}+f_{100}\right)
\end{aligned}
$$

$L_{\mathrm{FIR}}$ was preferentially chosen instead of $L_{\mathrm{IR}}$ because the 1.9 Jy survey includes fluxes from the Point Source Catalog (PSC), which has a low sensitivity in the midinfrared bands, $12 \mu \mathrm{m}$ and $25 \mu \mathrm{m}$, used in the determination of $L_{\mathrm{IR}}$.

With all the criteria above-mentioned, we found the 24 galaxies listed in Table 1. The optical positions in Cols. 2-3 are given by Strauss et al. (1992); the redshifts in Col. 4 have been calculated from our spectrophotometric data, when available. For systems made of close merging disks, the mean redshift is indicated, whereas in loose interacting systems, the quoted redshift is that of the nearest galaxy from the IRAS position. The mid and far infrared fluxes from the IRAS Faint Source Catalog (FSC) version 2, more sensitive than the PSC initially used by Strauss et al. (1992), are given in Cols. 5-8. The infrared, $L_{\mathrm{IR}}$, and far-infrared luminosities, $L_{\mathrm{FIR}}$, have been recalculated with our new redshifts and with the FSC fluxes (Cols. 9-10). Figure 1 and Fig. 2 present the redshift and luminosity distributions. Redshifts, $z$, range between 0.04 and 0.13; with our selection criteria, the SULIRG sample is complete up to $z=0.1$. The most luminous object is IRAS $20100-4156$, with $L_{\mathrm{IR}}=2.210^{12} L_{\odot}$.

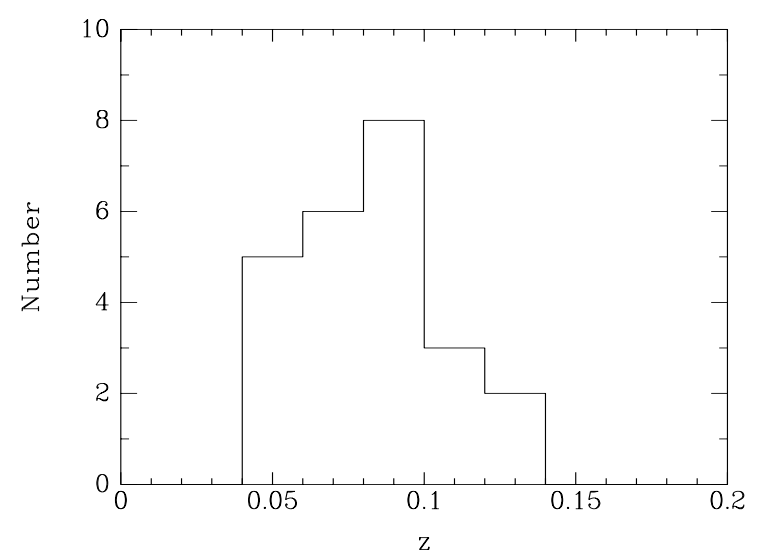

Fig. 1. Redshift distribution of SURILGs

The SULIRG sample includes the list of ULIRGs compiled by Melnick \& Mirabel (1990). The more northern objects are also present in the catalog of Murphy et al. (1996) (however these authors present only imaging data); three galaxies belong to the IRAS Bright 
Table 1. IRAS Properties of southern ultraluminous infrared galaxies

\begin{tabular}{|c|c|c|c|c|c|c|c|c|c|}
\hline IRAS name & $\mathrm{RA}$ & DEC & $z$ & & C flux & nsities & Jy) & $\log \left(L_{\mathrm{FIR}} / L_{\odot}\right)$ & $\log \left(L_{\mathrm{IR}} / L_{\odot}\right)$ \\
\hline & B1950 & B1950 & & $12 \mu \mathrm{m}$ & $25 \mu \mathrm{m}$ & $60 \mu \mathrm{m}$ & $100 \mu \mathrm{m}$ & & \\
\hline (1) & $(2)$ & (3) & (4) & $(5)$ & $(6)$ & (7) & (8) & (9) & $(10)$ \\
\hline $00199-7426$ & 001956.4 & -742610 & 0.0963 & 0.11 & 0.33 & 4.16 & 6.42 & 12.02 & 12.08 \\
\hline $05189-2524$ & 051858.6 & -252439 & 0.0427 & 0.73 & 3.44 & 13.67 & 11.36 & 11.82 & 12.02 \\
\hline $06035-7102$ & 060334.6 & -710258 & 0.0794 & 0.12 & 0.57 & 5.13 & 5.65 & 11.91 & 12.00 \\
\hline $06206-6315$ & 062040.3 & -631551 & 0.0920 & 0.07 & 0.29 & 3.96 & 4.58 & 11.92 & 11.98 \\
\hline $09061-1248$ & 090611.7 & -124848 & 0.0738 & 0.12 & 0.19 & 3.63 & 5.32 & 11.75 & 11.81 \\
\hline $09111-1007$ & 091111.1 & -100701 & 0.0537 & 0.17 & 0.47 & 7.08 & 11.06 & 11.80 & 11.86 \\
\hline $11095-0238$ & 110930.2 & -023801 & 0.1065 & 0.13 & 0.42 & 3.25 & 2.53 & 11.89 & 12.02 \\
\hline $14348-1447$ & 143452.3 & -144724 & 0.0823 & 0.14 & 0.49 & 6.87 & 7.07 & 12.06 & 12.13 \\
\hline $14378-3651$ & 143753.4 & -365143 & 0.0682 & 0.12 & 0.52 & 6.19 & 6.34 & 11.87 & 11.94 \\
\hline $15462-0450$ & 154617.6 & -045026 & 0.0998 & 0.13 & 0.45 & 2.92 & 3.00 & 11.83 & 11.97 \\
\hline $16090-0139$ & 160904.3 & -013927 & 0.1334 & 0.09 & 0.26 & 3.61 & 4.87 & 12.17 & 12.23 \\
\hline $17208-0014$ & 172048.2 & -001417 & 0.0429 & 0.20 & 1.66 & 31.14 & 34.90 & 12.22 & 12.25 \\
\hline $19254-7245$ & 192527.8 & -724539 & 0.0617 & 0.22 & 1.24 & 5.48 & 5.79 & 11.74 & 11.91 \\
\hline $20046-0623$ & 200439.2 & -062310 & 0.0845 & 0.09 & 0.22 & 3.23 & 3.91 & 11.77 & 11.85 \\
\hline $20087-0308$ & $2008 \quad 46.4$ & -030852 & 0.1055 & 0.13 & 0.24 & 4.70 & 6.54 & 12.12 & 12.18 \\
\hline $20100-4156$ & 201005.8 & -415639 & 0.1298 & 0.13 & 0.34 & 5.23 & 5.16 & 12.27 & 12.34 \\
\hline $20414-1651$ & 204128.3 & -165112 & 0.0870 & 0.65 & 0.35 & 4.36 & 5.25 & 11.92 & 12.14 \\
\hline $20551-4250$ & 205509.3 & -425037 & 0.0426 & 0.28 & 1.91 & 12.78 & 9.95 & 11.78 & 11.90 \\
\hline $21130-4446$ & 211300.5 & $-44 \quad 4614$ & 0.0929 & 0.07 & 0.16 & 3.11 & 3.78 & 11.83 & 11.88 \\
\hline $21504-0628$ & 215028.4 & -062855 & 0.0775 & 0.09 & 0.39 & 3.48 & 2.89 & 11.69 & 11.80 \\
\hline $22491-1808$ & 224909.5 & -180818 & 0.0777 & 0.12 & 0.55 & 5.44 & 4.45 & 11.89 & 11.98 \\
\hline $23128-5919$ & 231250.6 & -591937 & 0.0445 & 0.24 & 1.59 & 10.80 & 10.99 & 11.77 & 11.88 \\
\hline $23230-6926$ & 232302.5 & -692647 & 0.1063 & 0.06 & 0.29 & 3.74 & 3.42 & 11.97 & 12.04 \\
\hline $23389-6139$ & 233859.4 & -613928 & 0.0932 & 0.06 & 0.24 & 3.63 & 4.26 & 11.89 & 11.95 \\
\hline
\end{tabular}

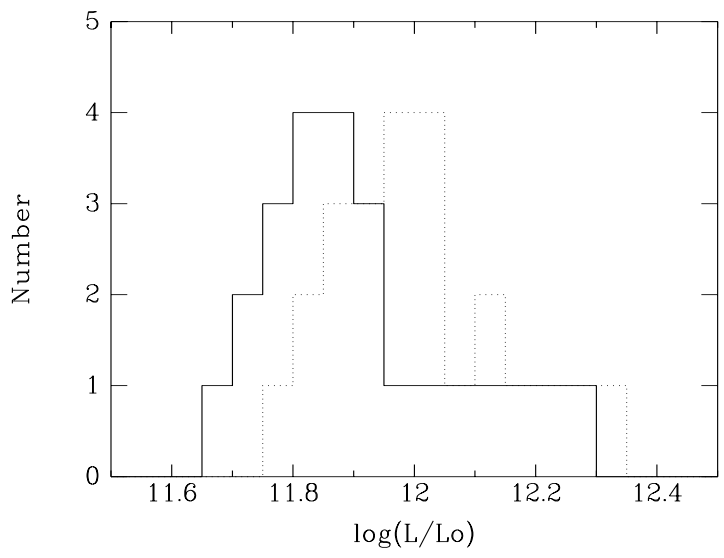

Fig. 2. Infrared (dotted) and far infrared (plain) luminosity distributions of SURILGs

Galaxy Sample (Soifer et al. 1987) and are therefore in common with the ULIRG sample of Sanders et al. (1988a). Some of the nearest objects have already been studied in details: IRAS $14348-1447$ (Sanders et al. 1988c), IRAS 19254-7245 ("The Super-Antennae", Mirabel et al.
1991; Colina et al. 1991), IRAS 20551 - 4251 (Johansson 1991) and IRAS 23128-5919 (Bergvall \& Johansson1985). Moreover Mirabel et al. (1988) have published CO measurements for 15 galaxies. One object, IRAS $17208-0014$, is known as an $\mathrm{OH}$ megamaser (Martin et al. 1989).

We have obtained optical CCD images for all the galaxies in our sample, but IRAS $05189-2524$, one of the BGS members, and optical spectra for 18 of them; 17 galaxies have also been imaged in the near infrared.

\section{Observations and data reduction}

Table 2 summarizes the observing parameters for each object in the sample: date of observations, telescope and instrument used, spatial or spectral resolution and exposure time.

\subsection{Optical imaging}

Optical images from the Melnick \& Mirabel (1990) sample have been obtained in September 1989 during the commissioning period of the NTT, at the European Southern Observatory. $R$ band observations were carried out with the EFOSC2 camera. Due to guiding and rotating 
Table 2. Observing parameters

\begin{tabular}{|c|c|c|c|c|c|c|c|c|c|c|c|c|}
\hline \multirow[b]{2}{*}{ IRAS name } & \multicolumn{4}{|c|}{ Optical imaging } & \multicolumn{4}{|c|}{ Optical spectroscopy } & \multicolumn{4}{|c|}{ Infrared imaging } \\
\hline & Date & Telescope & $\begin{array}{l}\text { Res. } \\
\text { "1/px } \\
(4)\end{array}$ & $\begin{array}{c}\text { Exp. } \\
\text { s } \\
(5) \\
\end{array}$ & Date & Telescope & $\begin{array}{c}\text { Res. }^{a} \\
\AA / \mathrm{px} \\
(8) \\
\end{array}$ & $\begin{array}{c}\text { Exp. }^{a} \\
\text { s } \\
(9) \\
\end{array}$ & Date & Telescope & $\begin{array}{l}\text { Res. } \\
\text { "1/px } \\
(12)\end{array}$ & $\begin{array}{c}\text { Exp. }^{b} \\
\text { s } \\
(13) \\
\end{array}$ \\
\hline $00199-7426$ & $07 / 94$ & $3.5 \mathrm{~m} \mathrm{NTT}$ & 0.27 & 300 & $12 / 95$ & $\mathrm{D} 1.5 \mathrm{~m} \mathrm{ESO}$ & 2.9 & 2700 & $02 / 96$ & $2.2 \mathrm{~m} \mathrm{ESO}$ & 0.51 & 480 \\
\hline $06035-7102$ & $08 / 89$ & $3.5 \mathrm{~m} \mathrm{NTT}$ & 0.25 & 20 & $07 / 89$ & 4 m CTIO & 5.7 & 600 & $02 / 96$ & $2.2 \mathrm{~m} \mathrm{ESO}$ & 0.51 & 480 \\
\hline $06206-6315$ & $08 / 89$ & $3.5 \mathrm{~m} \mathrm{NTT}$ & 0.25 & 60 & $07 / 89$ & 4 m CTIO & 5.7 & 600 & - & - & - & - \\
\hline $09061-1248$ & $02 / 93$ & $3.5 \mathrm{~m} \mathrm{NTT}$ & 0.44 & 60 & $02 / 93$ & $3.5 \mathrm{~m} \mathrm{NTT}$ & 4.5 & 1800 & $02 / 96$ & $2.2 \mathrm{~m} \mathrm{ESO}$ & 0.51 & 480 \\
\hline $09111-1007$ & $02 / 93$ & $3.5 \mathrm{~m} \mathrm{NTT}$ & 0.44 & 60 & $02 / 92$ & $3.5 \mathrm{~m} \mathrm{NTT}$ & 4.5 & 1800 & $02 / 96$ & $2.2 \mathrm{~m} \mathrm{ESO}$ & 0.51 & 480 \\
\hline $11095-0238$ & $02 / 93$ & $3.5 \mathrm{~m} \mathrm{NTT}$ & 0.44 & 120 & $07 / 89$ & 4 m CTIO & 5.7 & 600 & $06 / 93$ & $2.2 \mathrm{~m} \mathrm{ESO}$ & 0.49 & 300 \\
\hline $14348-1447$ & $08 / 89$ & $3.5 \mathrm{~m} \mathrm{NTT}$ & 0.25 & 120 & - & - & - & - & - & - & - & - \\
\hline $14378-3651$ & $08 / 89$ & $3.5 \mathrm{~m} \mathrm{NTT}$ & 0.25 & 180 & $07 / 88$ & $4 \mathrm{~m}$ CTIO & 5.7 & 1200 & $06 / 93$ & $2.2 \mathrm{~m} \mathrm{ESO}$ & 0.27 & 300 \\
\hline $15462-0450$ & $07 / 94$ & $3.5 \mathrm{~m} \mathrm{NTT}$ & 0.27 & 600 & $02 / 93$ & $3.5 \mathrm{~m} \mathrm{NTT}$ & 3.7 & 900 & $06 / 93$ & $2.2 \mathrm{~m} \mathrm{ESO}$ & 0.27 & 300 \\
\hline $16090-0139$ & $07 / 94$ & $3.5 \mathrm{~m} \mathrm{NTT}$ & 0.27 & 300 & - & - & - & - & - & - & - & - \\
\hline $17208-0014$ & $08 / 89$ & $3.5 \mathrm{~m} \mathrm{NTT}$ & 0.25 & 130 & $07 / 89$ & $4 \mathrm{~m} \mathrm{CTIO}$ & 5.7 & 1200 & $06 / 93$ & $2.2 \mathrm{~m} \mathrm{ESO}$ & 0.49 & 300 \\
\hline $19254-7245$ & $06 / 92$ & $3.6 \mathrm{~m} \mathrm{ESO}$ & 0.81 & 600 & $07 / 88$ & $4 \mathrm{~m} \mathrm{CTIO}$ & 5.7 & 1200 & $06 / 92$ & $2.2 \mathrm{~m} \mathrm{ESO}$ & 0.49 & 900 \\
\hline $20046-0623$ & $07 / 94$ & $3.5 \mathrm{~m} \mathrm{NTT}$ & 0.27 & 300 & - & - & - & - & - & - & - & - \\
\hline $20087-0308$ & $07 / 94$ & $3.5 \mathrm{~m} \mathrm{NTT}$ & 0.27 & 300 & $07 / 89$ & $4 \mathrm{~m}$ CTIO & 5.7 & 1200 & $06 / 93$ & $2.2 \mathrm{~m} \mathrm{ESO}$ & 0.49 & 300 \\
\hline $20100-4156$ & $08 / 89$ & $3.5 \mathrm{~m} \mathrm{NTT}$ & 0.25 & 180 & $07 / 88$ & $4 \mathrm{~m}$ CTIO & 5.7 & 1200 & $06 / 92$ & $2.2 \mathrm{~m} \mathrm{ESO}$ & 0.49 & 300 \\
\hline $20414-1651$ & $08 / 89$ & $3.5 \mathrm{~m} \mathrm{NTT}$ & 0.25 & 180 & $07 / 89$ & 4 m CTIO & 5.7 & 600 & $06 / 92$ & $2.2 \mathrm{~m} \mathrm{ESO}$ & 0.49 & 300 \\
\hline $20551-4250$ & $08 / 89$ & $3.5 \mathrm{~m} \mathrm{NTT}$ & 0.25 & 180 & $07 / 88$ & 4 m CTIO & 5.7 & 600 & $06 / 92$ & $2.2 \mathrm{~m} \mathrm{ESO}$ & 0.49 & 300 \\
\hline $21130-4446$ & $08 / 89$ & $3.5 \mathrm{~m} \mathrm{NTT}$ & 0.25 & 180 & $07 / 89$ & $4 \mathrm{~m}$ CTIO & 5.7 & 720 & $06 / 92$ & $2.2 \mathrm{~m} \mathrm{ESO}$ & 0.27 & 300 \\
\hline $21504-0628$ & $07 / 94$ & $3.5 \mathrm{~m} \mathrm{NTT}$ & 0.27 & 300 & - & - & - & - & - & - & - & - \\
\hline $22491-1808$ & $06 / 92$ & $3.6 \mathrm{~m} \mathrm{ESO}$ & 0.81 & 600 & - & - & - & - & - & - & - & - \\
\hline $23128-5919$ & $08 / 89$ & $3.5 \mathrm{~m} \mathrm{NTT}$ & 0.25 & 180 & $07 / 89$ & $4 \mathrm{~m} \mathrm{CTIO}$ & 5.7 & 600 & $06 / 92$ & $2.2 \mathrm{~m} \mathrm{ESO}$ & 0.49 & 300 \\
\hline $23230-6926$ & $07 / 94$ & $3.5 \mathrm{~m} \mathrm{NTT}$ & 0.27 & 300 & $07 / 88$ & $4 \mathrm{~m} \mathrm{CTIO}$ & 5.7 & 1200 & $07 / 94$ & $2.2 \mathrm{~m} \mathrm{ESO}$ & 0.27 & 900 \\
\hline $23389-6139$ & $07 / 94$ & $3.5 \mathrm{~m} \mathrm{NTT}$ & 0.27 & 300 & $07 / 89$ & $4 \mathrm{~m}$ CTIO & 5.7 & 900 & $07 / 94$ & $2.2 \mathrm{~m} \mathrm{ESO}$ & 0.49 & 200 \\
\hline
\end{tabular}

${ }^{a}$ data for LR spectra. ${ }^{b}$ for $K$ band.

problems, the exposure time had to be limited to 2 min. Optical data have been complemented in June 1992, February 1993 and July 1994 with images obtained using the EFOSC camera on the ESO $3.6 \mathrm{~m}$, and the EMMI camera on the NTT. They have been reduced with standard procedures using the IRAF CCDRED package.

Figure 3 presents for each system in the sample (except IRAS 05189 - 2524; an image of this object can be found in Sanders et al. 1988a), a greyscale large field image and a contour map of the central region in a logarithm scale. The images obtained during the 1989 run had already been published in Melnick \& Mirabel (1990). They have however been reprocessed and reproduced here to emphasize the central regions; they also have been rotated so that the orientation is the same for all objects. For comparison purposes, an angular and linear scale are indicated in each image.

The photometry has been done with the APPHOT package within IRAF. The derived $R$ band magnitudes can be found in Table 3. Since, during the 1989 and 1992 runs, no standard photometric stars had been observed, we could only obtain a rough estimate of the $R$ magnitude (with an error of $\sim 0.3 \mathrm{mag}$ ) by calibrating the data with published magnitudes. The 1993 and 1994 observations were done under photometric conditions, and Landolt standards stars have been observed (Landolt 1992). The error for these objects is typically $0.1 \mathrm{mag}$. A polygonal aperture was used to derive the total magnitude because of the peculiar morphology of ULIRGs. The limiting isophote corresponds roughly to $25 \mathrm{mag} \operatorname{arcsec}^{-2}$. If several objects are present in the IRAS field, the data are given for each of them. For multi nucleated systems, the magnitude of the whole system and of the nuclei are mentioned. For these, a circular aperture was used. The corresponding radius is indicated in Col. 3 of Table 3.

\subsection{Near infrared imaging}

Near infrared imaging was mainly carried out in June 1992 and June 1993 with the IRAC 2 camera, installed on the ESO/MPI 2.2-meter telescope. Additional observations with an upgraded version of the instrument, IRAC 2B, were done in July 1994 and February 1996. The detector was a $256 \times 256 \mathrm{HgCdTe}$ NICMOS 3 array. According to the seeing conditions, we have either used the 0.27 or $0.5 \mathrm{arcsec} /$ pixel objectives with the $J, H$ and $K$ filters. The individual exposures ranged between $1 \mathrm{~s}$ and $20 \mathrm{~s}$ depending on the filter and on the background level. The number of individual frames taken for one position was adjusted to reach an integration time of $1 \mathrm{~min}$. For sky acquisition the telescope was offset by typically $30^{\prime \prime}$ in each direction around the central position, with most of the time the object still in the field, so that no observing time was lost. The images were sky subtracted using a sky obtained by median filtering the images of the different positions. The final frame was obtained by a shift and add method on the individual images. To increase the 
signal to noise, the images were smoothed with a flattopped rectangular kernel. The total on-source integration time for the $K$ band is given in Table 2 .

The photometry has been done with circular apertures centered on each nucleus. For flux calibration, faint stars from the IRIS list of SAAO standard stars (Carter $\&$ Meadows 1995) have been observed. However, during our 1992 run, the weather conditions have not been photometric. Nevertheless, for the few objects that we have observed during both our 1992 and 1993 runs, we did not find any significant discrepancy within the data. The magnitude difference is less than 0.1 mag. Photometric data are given in Table 3 .

Figure 4 presents the $K$ band calibrated contour maps of the 17 galaxies that we have observed. The angular and linear scales are indicated. Contour maps of the three SULIRGs belonging to the BGS can be found in Carico et al. (1988) and Murphy et al. (1996) have published an uncalibrated $K$ image of IRAS $20046-0623$.

\subsection{Optical spectroscopy}

Low and high resolution spectra have mostly been obtained in July 1988 and July 1989 during two observing runs with the 4-meter telescope at Cerro Tololo InterAmerican Observatory. Two gratings were used, one covering the spectral range $4400-7700 \AA$ with a pixel resolution of $5.7 \AA$; the other covering $6500-7500 \AA$ with a pixel resolution of $1.8 \AA$. The spatial resolution along the slit was 0 "' $4 / \mathrm{px}$. Its width was 1 "' 3 . For three objects, we have obtained in February 1994 low resolution spectra with the EMMI instrument installed on the ESO NTT. The grism used had a pixel resolution of $4.5 \AA$. The spectrum of IRAS 00199 - 7426 was taken in December 1995, with the DFOSC instrument installed at the Danish $1.5 \mathrm{~m}$ telescope at La Silla Observatory. In total, spectroscopic data for 18 galaxies have been collected. Three more, IRAS 05189 - 2524, IRAS 14348 - 1447 and IRAS $22491-1808$ have spectra published in Sanders et al. (1988a).

The data reduction was carried out with the LONGSLIT package within IRAF. The spectra of the galactic nuclei were extracted from the $2 \mathrm{D}$ spectra. The angular size over which the extraction was done depended on the distance of the objects, and on the nuclei separation for interacting objects. It ranged between $2-5^{\prime \prime}$. Standard stars from the list of Stone \& Baldwin (1983) have been observed for flux calibration. The calibrated low and high resolution spectra are displayed in Fig. 5. For the galaxies observed with the NTT, a close-up of the low resolution spectrum in the red wavelength range is shown instead of the high resolution spectrum. The spectrophotometry of the main optical, low resolution lines, is presented in Table 4 . The fluxes and equivalent widths have been directly measured with the SPLOT task. The flux uncertainty, estimated from successive line measurements, is typically $10 \%$, for bright lines with fluxes higher than $1.010^{-15} \mathrm{erg} \mathrm{cm}^{-2} \mathrm{~s}^{-1}$. It may be higher in the case of the $\mathrm{H}_{\beta}$ line, strongly contaminated in some galaxies by stellar absorption. It is indicated in Col. 3, whether the $\mathrm{H}_{\beta}$ line is seen in emission, in absorption, or in emission within an absorption line. Another uncertainty affecting the spectrophotometry comes from the differential atmospheric refraction (Filippenko 1982). For interacting galaxies, the slit was preferentially put along the double nuclei, and therefore was not oriented along the parallactic angle. Since most of our objects $(60 \%)$ have been observed with airmasses below 1.4, the differential refraction within our spectral range was less than 0.5 , which is small compared to our slit width. For $15 \%$ of them however, observed with an airmass greater than 2 , and for which the differential refraction might have been, in the worst cases, as high as $1^{\prime \prime} 0$, spectrophotometric errors might have been significant. In Table 4, uncertain fluxes and equivalent width measurements, with errors greater than $10 \%$, are marked with a ":".

\section{Data analysis}

\subsection{Optical and near-infrared morphology}

Table 5 summarizes the morphological information on the SULIRGs, based on our optical and infrared observations. Data from the literature have been added. The projected angular and, in parentheses, the linear separation between the nuclei of interacting galaxies is indicated in Col. 4. The infrared images have preferentially been used to measure them. For objects with a single nucleus, we have estimated an upper limit calculated from the FWHM of stars in the field. In loose pairs, we also give, within brackets, the angular and linear distance of the companion. When available the velocity difference between the interacting partners is indicated in Col. 5. The velocities have been determined using the mean redshift of $[\mathrm{OI}]_{\lambda 6300}, \mathrm{H}_{\alpha},[\mathrm{NII}]_{\lambda 6584}$ and $[\mathrm{SII}]_{\lambda 6717,6731}$ high resolution lines.

In our sample of 24 systems, $13(54 \%)$ are close interacting galaxies (with a linear separation less than $12 \mathrm{kpc}$ ) or merging objects, having two nuclei enshrouded in a common envelope. $5(21 \%)$ have a single nucleus both in the optical and in the infrared but exhibit typical tidal tails of mergers. 3 (12.5\%) do not show direct evidences of interaction but their slightly disturbed morphology for instance, an asymmetry in their elliptical isophotes (IRAS 14378 - 3651), an eccentric (IRAS 16090 - 0139) or elongated (IRAS 23230 - 6926) nucleus - suggest that they are the remnants of complete mergers. Their tidal tails would have had time to dissipate since the collision or they are too far and faint to be visible. Finally, $3(12.5 \%)$ objects have an ambiguous nature, discussed hereafter.

A clear proof of a merger origin for these objects could undoubtedly be obtained in disentangling their nucleus. Most of the galaxies in the sample of Sanders et al. 
Table 3. Optical and near infrared photometry of SULIRGs

\begin{tabular}{|c|c|c|c|c|c|c|c|c|}
\hline \multirow[b]{2}{*}{ IRAS name } & \multicolumn{3}{|c|}{ Optical } & \multicolumn{5}{|c|}{ Infrared } \\
\hline & $\begin{array}{c}R \\
\operatorname{mag} \\
(2)\end{array}$ & $\begin{array}{c}\text { Apert } \\
(3) \\
(3)\end{array}$ & (4) & $\begin{array}{c}J \\
\operatorname{mag} \\
(5)\end{array}$ & $\begin{array}{c}H \\
\operatorname{mag} \\
(6)\end{array}$ & $\begin{array}{c}K \\
\text { mag } \\
(7)\end{array}$ & $\begin{array}{c}\text { Apert } \\
(8)\end{array}$ & Ref \\
\hline $00199-7426$ & 15.0 & tot & $\mathrm{a}$ & 14.1 & 13.2 & 12.5 & 5.0 & $\mathrm{a}$ \\
\hline $06035-7102$ & 15.6 & tot & $\mathrm{b}$ & 13.3 & 12.6 & 12.0 & 13 & $\mathrm{a}$ \\
\hline $06035-7102 \mathrm{~W}$ & 16.8 & 3 & $\mathrm{~b}$ & 14.9 & 14.2 & 13.6 & 3 & $\mathrm{a}$ \\
\hline $06035-7102 \mathrm{E}$ & 17.0 & 3 & $\mathrm{~b}$ & 14.4 & 13.6 & 12.8 & 3 & $\mathrm{a}$ \\
\hline $06206-6315$ & 16.3 & tot & $\mathrm{b}$ & - & - & - & - & - \\
\hline $09061-1248$ & 15.8 & tot & $\mathrm{a}$ & 14.0 & 13.2 & 12.6 & 7.5 & $\mathrm{a}$ \\
\hline $09061-1248 \mathrm{~S}$ & 17.0 & 2.5 & $\mathrm{a}$ & 14.9 & 14.0 & 13.2 & 2.5 & $\mathrm{a}$ \\
\hline $09061-1248 \mathrm{~N}$ & 17.3 & 2.5 & $\mathrm{a}$ & 15.5 & 14.7 & 14.2 & 2.5 & $\mathrm{a}$ \\
\hline $09111-1007 \mathrm{~W}$ & 15.7 & tot & $\mathrm{a}$ & 13.3 & 12.4 & 11.9 & 15 & $\mathrm{a}$ \\
\hline $09111-1007 \mathrm{E}$ & 14.8 & tot & $\mathrm{a}$ & 13.0 & 12.4 & 12.0 & 15 & $\mathrm{a}$ \\
\hline $11095-0238$ & 16.8 & tot & $\mathrm{a}$ & 16.1 & 15.6 & 14.7 & 5 & $\mathrm{a}$ \\
\hline $14348-1447$ & 15.8 & tot & $\mathrm{b}$ & 15.0 & 14.1 & 13.3 & 5 & $\mathrm{~b}$ \\
\hline $14348-1447 \mathrm{~S}$ & 16.9 & 2.5 & $\mathrm{~b}$ & 15.6 & 14.7 & 13.7 & 2.5 & $\mathrm{~b}$ \\
\hline $14348-1447 \mathrm{~N}$ & 18.2 & 2.5 & $\mathrm{~b}$ & 16.1 & 15.1 & 14.3 & 2.5 & $\mathrm{~b}$ \\
\hline $14378-3651$ & 16.3 & tot & $\mathrm{b}$ & 14.5 & 13.9 & 12.8 & 5 & $\mathrm{a}$ \\
\hline $15462-0450 / \mathrm{N}$ & 16.6 & tot & $\mathrm{a}$ & 14.8 & 13.6 & 12.2 & 5 & $\mathrm{a}$ \\
\hline $15462-0450 / \mathrm{S}$ & 17.3 & tot & $\mathrm{a}$ & - & - & - & - & - \\
\hline $16090-0139$ & 16.7 & tot & $\mathrm{a}$ & - & - & - & - & $\mathrm{c}$ \\
\hline $17208-0014$ & 14.8 & tot & $\mathrm{a}$ & 14.0 & 12.9 & 12.4 & 13 & $\mathrm{a}$ \\
\hline $19254-7245$ & 14.7 & - & $\mathrm{b}$ & 14.0 & 12.7 & 11.6 & 15 & $\mathrm{a}$ \\
\hline $19254-7245 / \mathrm{S}$ & 15.8 & 8 & $\mathrm{~b}$ & 14.7 & 13.5 & 12.2 & 8 & $\mathrm{a}$ \\
\hline $19254-7245 / \mathrm{N}$ & 16.2 & 8 & $\mathrm{~b}$ & 15.4 & 14.3 & 13.1 & 8 & $\mathrm{a}$ \\
\hline $20046-0623$ & 16.2 & tot & $\mathrm{a}$ & - & - & - & - & c \\
\hline $20088-0308$ & 15.9 & tot & $\mathrm{a}$ & 14.7 & 13.6 & 12.8 & 5 & $\mathrm{a}$ \\
\hline $20100-4156$ & 16.5 & tot & $\mathrm{b}$ & 15.0 & 14.3 & 13.7 & 7.5 & $\mathrm{a}$ \\
\hline $20100-4156 / \mathrm{S}$ & 18.2 & 1.2 & $\mathrm{~b}$ & 16.6 & 15.6 & 15.8 & 1.2 & $\mathrm{a}$ \\
\hline $20100-4156 / \mathrm{N}$ & 18.6 & 1.2 & $\mathrm{~b}$ & 17.4 & 16.7 & 16.7 & 1.25 & $\mathrm{a}$ \\
\hline $20414-1651$ & 16.9 & tot & $\mathrm{a}$ & 15.4 & 14.2 & 13.3 & 5 & $\mathrm{a}$ \\
\hline $20551-4250$ & 14.2 & 31 & $\mathrm{a}$ & 13.4 & 12.2 & 12.0 & 13 & $\mathrm{a}$ \\
\hline $21130-4446$ & 16.1 & tot & $\mathrm{b}$ & 15.3 & 14.1 & 13.5 & 9 & $\mathrm{a}$ \\
\hline $21130-4446 / \mathrm{S}$ & 17.9 & 1.5 & $\mathrm{~b}$ & 17.1 & 16.0 & 15.3 & 1.5 & $\mathrm{a}$ \\
\hline $21130-4446 / \mathrm{N}$ & 17.6 & 1.5 & $\mathrm{~b}$ & 16.6 & 15.3 & 14.8 & 1.5 & $\mathrm{a}$ \\
\hline $21504-0628$ & 15.6 & tot & $\mathrm{a}$ & - & - & - & - & - \\
\hline $22491-1808$ & 15.4 & tot & $\mathrm{b}$ & 14.7 & 14.0 & 13.6 & 5 & $\mathrm{~b}$ \\
\hline $23128-5919$ & 14.1 & tot & $\mathrm{b}$ & 13.4 & 12.3 & 11.8 & 10 & $\mathrm{a}$ \\
\hline $23128-5919 / \mathrm{S}$ & 15.7 & 2.5 & $\mathrm{~b}$ & 14.3 & 13.4 & 12.6 & 2.5 & $\mathrm{a}$ \\
\hline $23128-5919 / \mathrm{N}$ & 15.9 & 2.5 & $\mathrm{~b}$ & 14.7 & 13.7 & 13.3 & 2.5 & $\mathrm{a}$ \\
\hline $23230-6926$ & 16.6 & tot & $\mathrm{a}$ & 15.4 & 14.2 & 13.6 & 5 & $\mathrm{a}$ \\
\hline $23389-6139$ & 16.4 & tot & $\mathrm{a}$ & 15.0 & 14.2 & 13.5 & 8 & $\mathrm{a}$ \\
\hline $23389-6139 / \mathrm{S}$ & 17.6 & 3 & $\mathrm{a}$ & 15.5 & 14.3 & 13.7 & 3 & $\mathrm{a}$ \\
\hline $23389-6139 / \mathrm{N}$ & 18.5 & 3 & $\mathrm{a}$ & 16.8 & 16.5 & 15.8 & 3 & $\mathrm{a}$ \\
\hline
\end{tabular}

References, Optical: a) NTT $(1993,94)$ photometric conditions b) 3.6 m (1992), NTT (1989), no standard stars had been taken. Rough estimate from published data (uncertainty: $\sim 0.3 \mathrm{mag}$ ) Infrared: a) ESO $2.2 \mathrm{~m}(1992,93,94,96)$ mixed weather conditions, b) Carico et al. (1990), c) Murphy et al. (1996), $K$ uncalibrated image. 
Table 4. Observed spectrophotometric low resolution data of SULIRGs

\begin{tabular}{|c|c|c|c|c|c|c|c|c|c|c|c|c|c|}
\hline \multirow[b]{2}{*}{ IRAS name } & \multicolumn{8}{|c|}{ Observed flux $\left(10^{-15} \mathrm{erg} \mathrm{cm}^{-2} \mathrm{~s}^{-1}\right)$} & \multicolumn{5}{|c|}{ Equivalent width $(\AA)$} \\
\hline & $(2)^{4}$ & $\begin{array}{c}I_{\beta} \\
61 \\
(3) \\
\end{array}$ & $\begin{array}{r}{[\mathrm{OIII}]} \\
4959 \\
(4) \\
\end{array}$ & $\begin{array}{r}{[\mathrm{OIII}]} \\
5007 \\
(5) \\
\end{array}$ & $\begin{array}{r}{[\mathrm{OI}]} \\
6300 \\
(6) \\
\end{array}$ & $\begin{array}{r}\mathrm{H}_{\alpha} \\
6563 \\
(7) \\
\end{array}$ & $\begin{array}{r}{[\mathrm{NII}]} \\
6584 \\
(8) \\
\end{array}$ & $\begin{array}{r}{[\mathrm{SII}]} \\
6717 / 31 \\
(9) \\
\end{array}$ & $\begin{array}{r}\mathrm{H}_{\beta} \\
4861 \\
(10) \\
\end{array}$ & $\begin{array}{r}\text { MgIb } \\
5173 \\
(11) \\
\end{array}$ & $\begin{array}{r}\text { NaID } \\
5890 \\
(12) \\
\end{array}$ & $\begin{array}{r}{[\mathrm{OI}]} \\
6300 \\
(13) \\
\end{array}$ & $\begin{array}{r}\mathrm{H}_{\alpha} \\
6563 \\
(14) \\
\end{array}$ \\
\hline $00199-7426$ & 1.00 & em & - & 1.51 & - & - & - & - & 5.68 & 0.53 & 8.98 & - & - \\
\hline 06035-7102/W & 1.62 & em & 1.35: & 2.72 & 2.39 & 21.66 & 9.92 & 11.07 & 4.64 & - & 0.35 & 4.76 & 48.01 \\
\hline 06035-7102/E & 2.17 & em & 1.55: & 2.04 & 1.12 & 18.54 & 7.66 & 5.15 & 5.98 & - & 1.01 & 3.75 & 40.46 \\
\hline 06206-6315 & 0.26 : & abs/em & - & 1.36 & - & 6.31 & 4.45 & 4.86 & 1.18: & 2.31 & 2.63 & - & 13.82 \\
\hline 09061-1248/S & 0.61: & abs/em & - & 0.53: & 0.71: & 7.09 & 4.51 & 3.94 & 3.44: & 0.55 & 1.13 & 2.75: & 26.54 \\
\hline $09061-1248 / \mathrm{N}$ & 1.95 & em & $0.25:$ & $0.74:$ & $0.75:$ & 12.67 & 8.01 & 4.74 & 9.29 & 0.18 & 2.23 & 2.63: & 43.44 \\
\hline 09111-1007/W & 0.14 & em & - & - & $0.32:$ & 6.70 & 4.87 & 1.60 & 2.38 & 0.49 & 15.50 & 2.28: & 43.35 \\
\hline 09111-1007/E & 4.10 & em & 5.64 & 16.15 & 2.97 & 43.73 & 32.54 & 15.63 & 17.98 & 0.49 & - & 9.10 & 138.80 \\
\hline $11095-0238$ & 1.35 & em & 0.72 & 2.29 & 1.46 & 13.38 & 8.15 & 6.66 & 10.76 & - & 1.48 & 17.38 & 88.43 \\
\hline 14378-3651 & - & - & 0.59: & 1.76 & 0.69: & 13.71 & 7.28 & 3.57 & - & 1.02 & 6.20 & 3.63: & 39.77 \\
\hline $15462-0450 / \mathrm{S}$ & 2.86 & em & $0.38:$ & 1.76 & - & 18.29 & 7.16 & 5.72 & 17.70 & 0.37 & 3.08 & - & 99.16 \\
\hline $15462-0450 / \mathrm{N} *$ & 19.45: & em & 10.67: & 16.17: & 1.04: & 55.09: & 23.20: & 5.66 & 49.63: & - & - & 2.67: & 120.40: \\
\hline $17208-0014$ & $0.59:$ & abs/em & - & 0.65: & 1.40 & 16.59 & 10.30 & 5.23 & 1.64: & 0.45 & 6.11 & 3.90 & 28.09 \\
\hline $19254-7245 / \mathrm{S}$ & 2.03 & $\mathrm{em}$ & 4.55 & 15.72 & 10.02 & 117.10 & - & 19.70 & 9.02 & - & 5.13 & 30.15 & 218.10 \\
\hline $19254-7245 / \mathrm{N}$ & $-0.37:$ & abs & - & - & - & 5.74 & 4.22 & 2.10 & 1.47: & 2.83 & 4.80 & 2.47 & 10.13 \\
\hline 20087-0308 & -0.93 & abs & - & - & - & 6.32 & 4.72 & 2.53 & 7.02 & 2.06 & 7.34 & 4.64 & 23.40 \\
\hline $20100-4156$ & 1.86 & em & 0.89: & 2.79 & 1.00 & 16.08 & 7.02 & 2.74 & 10.51 & 0.11 & 3.06 & 7.93 & 56.82 \\
\hline 20414-1651 & 0.93: & $\mathrm{abs} / \mathrm{em}$ & 0.71: & 1.33 & 0.86 : & 9.76 & 4.73 & 4.21 & 6.46: & 1.32 & 2.19 & $6.46:$ & 41.18 \\
\hline $20551-4250$ & 6.39 & em & 2.57 & 6.78 & 4.58 & 61.44 & 24.47 & 23.71 & 8.70 & 1.12 & 0.99 & 4.77 & 42.91 \\
\hline $21130-4446$ & 9.68 & em & 4.27 & 13.11 & 1.09 & 45.05 & 11.57 & 13.63 & 29.75 & 0.87 & - & 5.56 & 130.00 \\
\hline $23128-5919 / \mathrm{N}$ & 8.86 & em & 5.54 & 14.48 & 3.09 & 82.99 & 19.76 & 23.72 & 20.45 & - & 1.31 & 5.91 & 115.30 \\
\hline $23128-5919 / \mathrm{S}$ & 13.32 & em & 13.60 & 40.09 & 4.54 & 93.35 & 29.50 & 25.61 & 20.68 & - & 1.64 & 8.35 & 131.70 \\
\hline $23230-6926$ & 1.08 & em & 1.04: & 1.71 & 1.10 & 13.51 & 5.83 & 6.32 & 8.06 & - & 2.32 & 5.89 & 71.67 \\
\hline 23389-6139 & $0.34:$ & em & 1.74 & 4.29 & - & 22.58 & - & 3.59 & $2.55:$ & 1.92 & 2.59 & 4.62 & 108.40 \\
\hline
\end{tabular}

* the line profiles show several line systems. Only the integrated values are given here.

(1988a), first thought to be single nucleated objects, appeared to have twin nuclei when observed in the infrared, a wavelength range less affected by obscuration, which is prominent in IRAS galaxies. However, apart from IRAS 22491 - 1808 (Carico et al. 1988), none of the SULIRGs, for which we have $R$ and $K$ images, show discrepant nuclear morphology between the two bands.

Compared to other ULIRGs, the ambiguous objects in our list appear as systems still in an early phase of an interaction process. Their companions are situated at a distance of $40-70 \mathrm{kpc}$ whereas most ultraluminous galaxies have disk-disk separation less than $10 \mathrm{kpc}$. One galaxy, IRAS 15462 - 0450, exhibits clear signs of tidal disruption; the two others, IRAS 09111 - 1007 and IRAS 20414-1651 seem to show an unperturbed morphology. More details on these systems are discussed in Sect. 5. The enhancement of the infrared luminosity in ULIRGs is generally explained by the heat of dust following either starbursts episodes in colliding gaseous clouds or the fueling of an active nucleus during the merging process. Such mechanisms imply a small distance between the colliding galaxies. The unexpected properties of our "ambiguous" objects could question this interpretation. However one cannot exclude the hypothesis that they are already merger remnants and that their companions do not take part in the ultraluminous phenomena.
The result that most, if not all, SULIRGs are facing a current interaction or have been subject to one in the past, agrees with previous analysis of ultraluminous galaxies (e.g. Sanders et al. 1988a; Clements et al. 1996a). The conclusion of Leech et al. (1994) who obtained, in their sample of 42 galaxies with $60 \mu \mathrm{m}$ luminosity greater than $10^{12} L_{\odot}$, a significant $17 \%$ fraction of apparently isolate systems, was recently questioned by Clements \& Baker (1996) who reobserved some of their systems and found signs of interactions in them.

\subsection{Spectral classification}

In this section, we try to classify the SULIRGs among the different classical spectral types: starburst (SB), LINERS (LI), Seyfert 2 (SII) and Seyfert 1 (SI).

While Seyfert 1 galaxies are easily recognizable thanks to their extremely large permitted emission lines, other objects are classified using diagnostic flux line ratios. As recommended by Veilleux \& Osterbrock (1987), to get rid as much as possible of extinction problems, we have used criteria involving ratios between nearby lines. The related three diagnostic diagrams, $[\mathrm{NII}]_{\lambda 6584} / \mathrm{H}_{\alpha}-$ $[\mathrm{OIII}]_{\lambda 5007} / \mathrm{H}_{\beta}, \quad[\mathrm{OI}]_{\lambda 6300} / \mathrm{H}_{\alpha}-[\mathrm{OIII}]_{\lambda 5007} / \mathrm{H}_{\beta}$ and $[\mathrm{SII}]_{\lambda 6717,6731} / \mathrm{H}_{\alpha}-[\mathrm{OIII}]_{\lambda 5007} / \mathrm{H}_{\beta}$, are shown in Fig. 6. To avoid line blending problems we have used, when available, the high resolution data. Care has been taken not to 
mix LR and HR lines. The fluxes have been corrected for Galactic extinction, and, if possible, for interstellar extinction, using a reddening curve parameterized by Miller \& Mathews (1972) as:

$A_{\lambda}=0.74 \lambda^{-1}-0.34$

and an absorption $A_{\mathrm{B}}$ derived from the Balmer decrement with the formula:

$A_{\mathrm{B}}=8.5 \log \left(\left(\mathrm{H}_{\alpha} / \mathrm{H}_{\beta}\right) / 3.0\right)$

$\mathrm{H}_{\alpha} / \mathrm{H}_{\beta}$ has been determined from the LR data. However the $\mathrm{LR} \mathrm{H}_{\alpha}$ line is strongly blended with the $[\mathrm{NII}]_{\lambda 6548}$ line. To take this effect into account the flux has been multiplied by the mean ratio between the $\mathrm{HR}$ and $\mathrm{LR} \mathrm{H}_{\alpha}$ fluxes. No attempt has been done to correct our data from intrinsic stellar absorption line. This causes an uncertainty in the $[\mathrm{OIII}]_{\lambda 5007} / \mathrm{H}_{\beta}$ ratio, which however is not too dramatic for the classification between SB and AGN (LINER or SII): the latter is more governed by the $[\mathrm{NII}]_{\lambda 6584} / \mathrm{H}_{\alpha}$ ratio. For clearness concerns, only the main galaxy or brightest nucleus, in systems with multiple components, is displayed in Fig. 6. Arrows correspond to galaxies with one undetermined line ratio.

Table 5 presents, in Col. 6 , the resulting adopted spectral classification for each system: the main galaxy or brightest nucleus in mergers, and below, either the faintest nucleus, or a possible interacting companion (in brackets). Since some galaxies do not have the same position in the different diagrams and others cannot be put into them, because some necessary lines were not detected, some rules had to be used, based on the appearance number of an object in a given spectral type area. The related convention for naming the spectral type is the following: " $a>b$ " means that two diagrams favor class " $a$ " and one class " $b$ ". " $a=b$ " indicates that the galaxy is on the border line. " $a$ ? $b$ " means that no choice between " $a$ " and " $b$ " can be done because of an undetermined flux ratio.

To do some statistics, we have given a weight to a spectral type $t$, proportional to the appearance number of a given galaxy in the locus of $t$, for the three diagnostic diagrams. An attempt to estimate an uncertainty associated with $t$ was made, based on the number of discrepancies of a galaxy position between the different diagrams. For cases with undetermined line ratios, an "undetermined type" column was charged. Applying these rules, we found, in our full sample, including two objects with data taken from the literature (see Table 4), that $25 \% \pm 10 \%$ of SULIRGs are starbursts, $28 \% \pm 7 \%$, LINERs and $23 \% \pm 3 \%$, Seyferts II. Besides, there is one Seyfert 1 galaxy in our sample (4\%), IRAS $15462-0450$. The properties of that peculiar object will be discussed in a future paper. For $20 \%$ of the galaxies, no spectral type could be given. Taking into account only galaxies having sufficient spectroscopic data for a reliable spectral determination, the distribution is the following: $29 \% \pm 11 \%$ for starbursts, $33 \% \pm 8 \%$ for LINERs, $27 \% \pm 3 \%$ for Seyferts II, $5 \% \pm 0$ for Seyferts I, and $6 \%$ for undetermined types.

The high fraction of AGNs among SULIRGs $(55 \% \pm$ $6 \%$ ) agrees with the trend found by Veilleux et al. (1995) of an increase in the proportion of AGNs with the infrared luminosity. Their sub-sample of ULIRGs extracted from the Extended BGS and IRAS Warm Galaxy Survey has a similar proportion of AGNs $(62 \%$, excluding the ambiguous objects), but a higher fraction of Seyfert 1 (15\%). Using low resolution spectra, Clements et al. (1996b) found that at least $35 \%$ of the ULIRGs in their sample are likely to contain an AGN-type central engine. However, when comparing statistics on spectral type coming from different studies, one has to take into account systematic biases, and among them, the way that individual nuclear spectra were extracted. Contamination by off-nuclear regions might significantly affect the determination of the spectral type. However, up to now, only very few studies have been done to quantify this bias (Kennicutt 1992; Lehnert \& Heckman 1994; Liu \& Kennicutt 1995), and none of them were actually devoted to AGN-like objects.

The question of the mechanism responsible for the infrared luminosity enhancement in ULIRGs has been widely addressed in the literature but no clear answer has yet emerged. Violent star forming episodes, nuclear activity, or a combination of both, have been proposed. In that context, one may wonder whether the high proportion of AGN nuclear spectral type apparently found among ultraluminous galaxies is an hint in favor of the second hypothesis. Some authors (Sanders et al. 1988a; Taniguchi et al. 1994) claim that the ultimate episode of the ultraluminous phase is the formation of a quasar buried in a merger remnant. However our candidates for complete mergers (IRAS 14378-3651, IRAS 20414-1651, IRAS $23230-6926$ ), do not show particularly active nuclear activity. The only Seyfert 1 in our sample may even not be a merger. Finally, one point should be emphasized. The ultraluminous galaxies are heavily obscured objects, as indicated by their high visual absorption and by radio continuum observations (Condon et al. 1991). As a result, the optical spectra may not reflect the properties of the actual nucleus. In that context, the determination of the fraction of AGNs found in ULIRGs from optical observations might be biased.

\section{Notes on individual objects}

A brief discussion is given here on some individual sources.

- IRAS 00199-7426: This galaxy lies in the sky close to SMC. The field is therefore crowded with SMC foreground stars. This contamination, added to a poor seeing, make it difficult to determine the morphology of the system. However, a central extended object, from 
which we got the spectrum displayed in Fig. 5, a tidal tail and two possible companions seem to be associated with IRAS $00199-7426$.

- IRAS 09111 - 1007: There are two galaxies in the field separated by a distance of $40^{\prime \prime}(42 \mathrm{kpc})$. Surprisingly, the IRAS position is centered on the western object, which is less luminous, in the optical, that the eastern disturbed spiral. Contrary to its companion, the infrared ultraluminous galaxy seems to have a regular spiral morphology. Since IRAS had a limited angular resolution, varying between $1^{\prime}$ and $5^{\prime}$, it is not clear whether in pairs, both galaxies are enhanced in the far infrared (Xu \& Sulentic 1991; Surace et al. 1993). In IRAS 09111 - 1007, the two galaxies (which are indeed at the same distance; the difference in velocity is only $300 \mathrm{~km} \mathrm{~s}^{-1}$ ) show strong optical lines. However, compared to the eastern object, the western one is extremely absorbed. $\mathrm{H}_{\beta}$ is invisible and there is a strong $\mathrm{Na}$ absorption line, which indicates a high dust content. Moreover, the radio continuum source associated with this source is stronger than the one associated with the companion (Karoji et al. 1986). Since there is a tight correlation between radio and far infrared fluxes (eg. Wunderlich et al. 1987), we can argue that most of the far infrared luminosity in this system comes from the western component.

- IRAS 11095-0238: It has two tidal tails, one prominent to the North with a condensation at its tip, and another fainter to the South-East. Both optical and infrared images show a single nucleus. This might be a merger in an advanced stage.

- IRAS $14378-3651$ : There is no obvious companion to this elliptical-like galaxy. Faint clumps can be found in the outskirts of the main body. It is likely to be a merger in a final stage.

- IRAS 15462 - 0450: The IRAS flux is concentrated on the northern spiral object of this loose interacting pair. The galaxy is highly disturbed. A long tail with bright condensations escapes to the North. An arc structure is seen to the West. Its Seyfert 1 spectrum shows strong iron FeII lines. Two line systems, separated by $\sim 1000 \mathrm{~km} \mathrm{~s}^{-1}$, are seen in the $\mathrm{H}_{\beta}$ and [OIII] line profiles. This object will be discussed in more details in a future paper.

- IRAS 16090 - 0139: This isolated object has an eccentric nucleus, also seen in the infrared (Murphy et al. 1996), which is an hint for a complete merger classification.

- IRAS 17208-0014: It has faint tidal tails and a disturbed morphology in the central region, with a bright nucleus surrounded by condensations (similar to HII regions) and absorption features. In the $K$ band however, the dust lanes disappear and only one nucleus shows up. It's luminosity profile follows the classical $r^{1 / 4}$ de Vaucouleurs law for ellipticals. This is a case where an elliptical galaxy is being formed after a diskdisk collision. Martin et al. (1989) have shown that this object is an $\mathrm{OH}$ megamaser.

- IRAS 19254 - 7245: This spectacular object, also known as "the Super-Antennae" has been discussed in details by Mirabel et al. (1991) and Colina et al. (1991).

- IRAS 20046 - 0623: No clear nucleus can be found in either interacting disk. Murphy et al. (1996) have shown that only the EW disk emits in $K$.

- IRAS 20087 - 0308: Two diffuse tails can be seen. The nucleus is clearly elongated to the East in the $R$ band. After a deconvolution by a gaussian PSF, with a Maximum Entropy method, two condensations separated by $2.6^{\prime \prime}(4.5 \mathrm{kpc})$ show up. In the infrared, however there is only a marginal detection at the position of the faintest source.

- IRAS 20100 - 4156: With an infrared luminosity of $2.210^{12} L_{\odot}$, it is the brightest source of our sample. It appears as a disk-disk collision. The northern disk seems to vanish in the $K$ band.

- IRAS 20414 - 1651: This is one of the ambiguous systems in the sample. Two main galaxies are visible, apparently separated by $41 \mathrm{kpc}$ (we do not have a redshift for the eastern object). The IRAS flux is concentrated on the western object. None of the galaxies show clear tidal disturbances. Faint objects can be seen in the vicinity of IRAS $20414-1651 / \mathrm{W}$.

- IRAS 20551 - 4151: discussed in details by Johansson (1991). Only one nucleus appears in the $R$ and $K$ bands of this merger.

- IRAS 22491 - 1808 (the so-called "South America Galaxy"): Carico et al. (1990) have shown, in the $K$ band image, two nuclei separated by $2^{\prime \prime}(3 \mathrm{kpc})$.

- IRAS 23128 - 5919 (the "Bat galaxy"): discussed in details by Bergvall \& Johansson (1985) and Johansson \& Bergvall (1988). The wings of this object are tidal features, whereas its eyes are the nuclei of the merging galaxies.

- IRAS 23230 - 6926: The nucleus seems to be elongated in the optical to the East. After deconvolution with a gaussian, one can infer a nuclei separation of 
$2.4^{\prime \prime}(4.1 \mathrm{kpc})$. The infrared image shows an elongated nucleus, but does not confirm that it is double.

- IRAS 23389 - 6139: Two nuclei are visible but one is at least twice as bright as the other in the optical. In $K$, the ratio is 10 .

\section{Conclusion}

We have obtained optical and near-infrared images, as well as low and high resolution optical spectra of a complete sample of Infrared Ultraluminous Galaxies in the Southern hemisphere (SULIRG). This database can be used for statistical studies and/or detailed works on individual objects. The general characteristics of the SULIRGs, briefly discussed in this paper, confirm the trends already noted from studies of other samples of ultraluminous IR galaxies: 1) most of these are interacting or merging systems and no instance of isolated normal objects has been found; 2) they have a high proportion of active nuclei. However there are exceptions to these general properties, since we found in our sample ultraluminous systems that consist of distant pairs, as well as complete mergers with no signs of nuclear activity.

Acknowledgements. We are grateful to Cecile Loup who obtained the spectrum of IRAS $00199-7426$. We wish to thank Dave Sanders and Dave Clements for helpful discussions, and our referee, N. Bergvall, for critical comments. This program has made use of the NASA/IPAC Extragalactic Database which is operated by the Jet Propulsion Laboratory, California Institute of Technology, under contract with the National Aeronautics and Space Administration.

\section{References}

Armus L., Heckman T.M., Miley G.K., 1990, ApJ 364, 471

Bergvall N., Johansson L., 1985, A\&A 149, 475

Carico D.P., Graham J.R., Matthews K., Wilson T.D., Soifer B.T., 1990, ApJ 349, L39

Carico D.P., Sanders D.B., Soifer B.T., Elias J.H., Matthews K., 1988, AJ 95, 356

Carter B.S., Meadows V.S., 1995, MNRAS 276, 734

Clements D.L., Baker A.C., 1996, ESO preprint 1180

Clements D.L., Sutherland W.J., McMahon R.G., Saunders W., 1996a, MNRAS 279, 477

Clements D.L., Sutherland W.J., Saunders W., et al., 1996, MNRAS 279, 459

Colina L., Lipari S., Macchetto F., 1991, ApJ 379, 113

Condon J.J., Huang Z.-P., Yin Q.F., Thuan T.X., 1991, ApJ 378,65
Filippenko A.V., 1982, PASP 94, 715

Johansson L., 1991, A\&A 241, 389

Johansson L., Bergvall N., 1988, A\&A 192, 81

Karoji H., Dennefeld M., Ukita N., 1986, A\&A 155, L3

Kennicutt R.C.J., 1992, ApJ 388, 310

Kim D.-C., 1995, Ph.D. Thesis, University of Hawaii

Landolt A.U., 1992, AJ 104, 340

Lawrence A., Walker D., Rowan-Robinson M., Leech K.J., Penston M.V., 1986, MNRAS 219, 687

Leech K.J., Rowan-Robinson M., Lawrence A., Hughes J.D., 1994, MNRAS 267, 253

Lehnert M.D., Heckman T.M., 1994, ApJL 426, L27

Liu C.T., Kennicutt R.C.J., 1995, ApJS 100, 325

Lonsdale C.J., Helou G., Good J.C., Rice W., 1985, Catalogued Galaxies and Quasars Observed in the IRAS Survey Jet Propulsion Laboratory, Pasadena, CA

Majewski S.R., Hereld M., Koo D.C., Illingworth G.D., Heckman T.M., 1993, ApJ 402, 125

Martin J.M., Le Squeren A.M., Bottinelli L., Gouguenheim L., Dennefeld M., 1989, A\&A 208, 39

Melnick J., Mirabel I.F., 1990, A\&A 231, L19

Miller J.S., Mathews W.G., 1972, ApJ 172, 593

Mirabel I.F., Lutz D., Maza J., 1991, A\&A 243, 367

Mirabel I.F., Sanders D.B., Booth R.S., Johansson L.E.B., Garay G., 1988, A\&A 206, L20

Moorwood A.F.M., 1996, Space Sci. Rev. 77, 303

Murphy T.W.J., Armus L., Matthews K., et al., 1996, AJ 111, 1025

Perault M., 1989, Ph.D. Thesis, University of Paris

Sanders D.B., Egami E., Lipari S., Mirabel I.F., Soifer B.T., 1996, ApJ (in press)

Sanders D.B., Mirabel I.F., 1996, ARA\&A 34, 749

Sanders D.B., Soifer B.T., Elias J.H., Matthews K., Madore B.F., 1988, ApJ 325, 74

Sanders D.B., Soifer B.T., Elias J.H., Neugebauer G., Matthews K., 1988, ApJ 328, L35

Sanders D.B., Soifer B.T., Scoville N.Z., 1988, Sci 239, 625

Soifer B.T., Sanders D.B., Neugebauer G., Danielson G.E., Lonsdale C.J., 1986, ApJ 303, L41

Soifer B.T., Sanders D.B., Neugebauer G., Madore B.F., Danielson G.E., 1987, ApJ 320, 238

Stone R.P.S., Baldwin J.A., 1983, MNRAS 204, 347

Strauss M.A., Huchra J.P., Davis M., et al., 1992, ApJS 83, 29

Surace J.A., Mazzarella J., Soifer B.T., Wehrle A.E., 1993, AJ 105,864

Taniguchi Y., Kawara K., Murayama T., Sato Y., 1994, ApJ 107,1668

Veilleux S., Kim D.-C., Sanders D.B., Mazzarella J.M., Soifer B.T., 1995, ApJS 98, 171

Veilleux S., Osterbrock D.E., 1987, ApJS 63, 295

Wunderlich E., Wielebinski R., Klein U., 1987, A\&AS 69, 487

Xu G., Sulentic J.W., 1991, ApJ 374, 407 
Table 5. Nature of the SULIRG systems

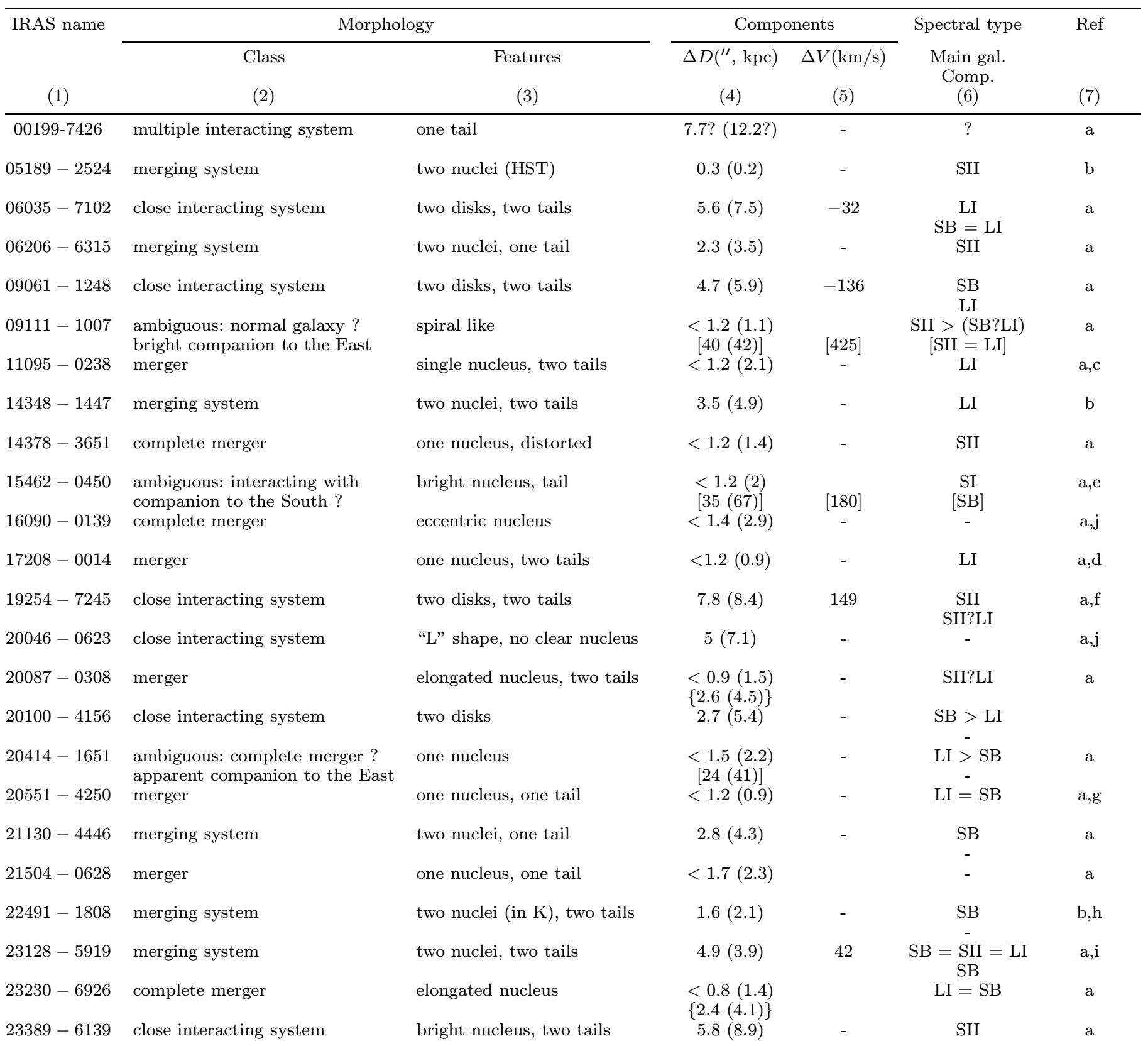

References a) this work, b) Sanders et al. (1988a), c) Armus et al. (1990), d) Martin et al. (1989), e) Duc \& Mirabel (1997), in preparation, f) Mirabel et al (1991), g) Johansson (1991), h) Carico et al. (1990), i) Bergvall \& Johansson (1985), Johansson \& Bergvall (1988), j) Murphy et al. (1996) abbreviations SB: Starburst, LI: LINER, S: Seyfert, $a$ ? $b$ : no criterion to determine between type $a$ and $b$, $a=b$ : divergent criteria, $a>b$ : two criteria favor type $a$ and one $b,[.$.$] : data for companion galaxy, \{.$.$\} : data for a second possible nucleus obtained after$ deconvolution of an elongated nucleus by a gaussian with a FWHM equal to the seeing. 


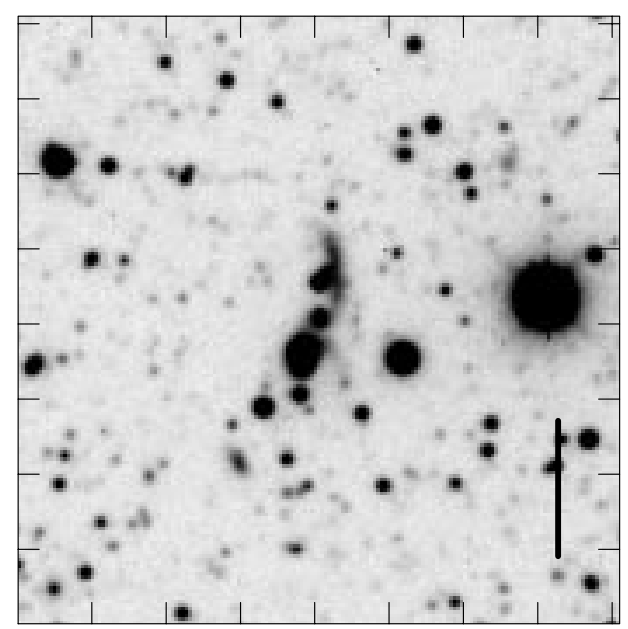

IRAS $00199-7426$

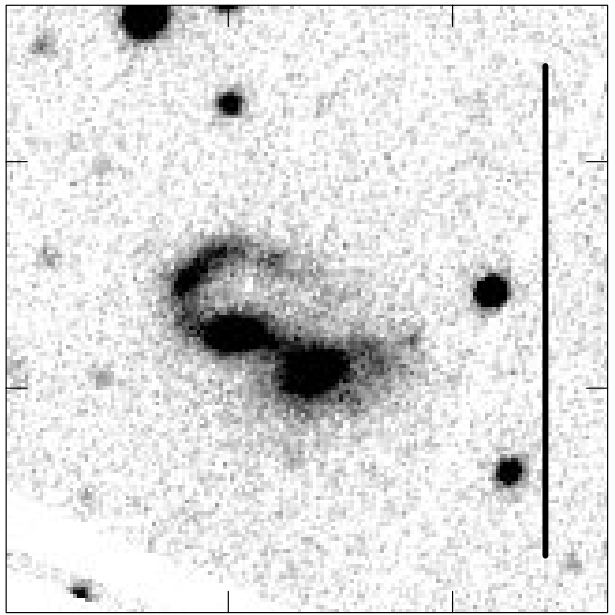

IRAS $06035-7102$

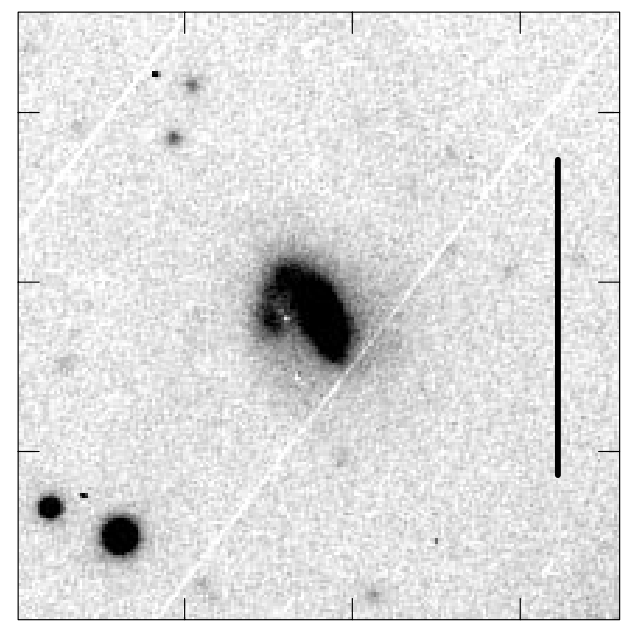

IRAS $06206-6315$

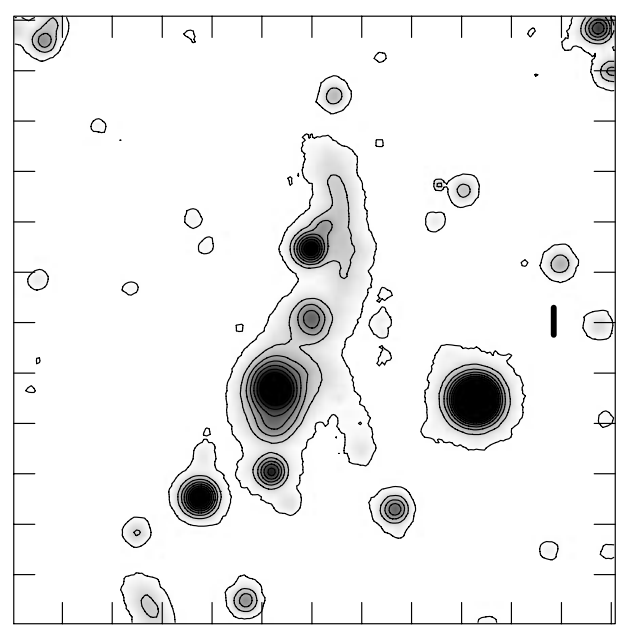

IRAS $00199-7426$

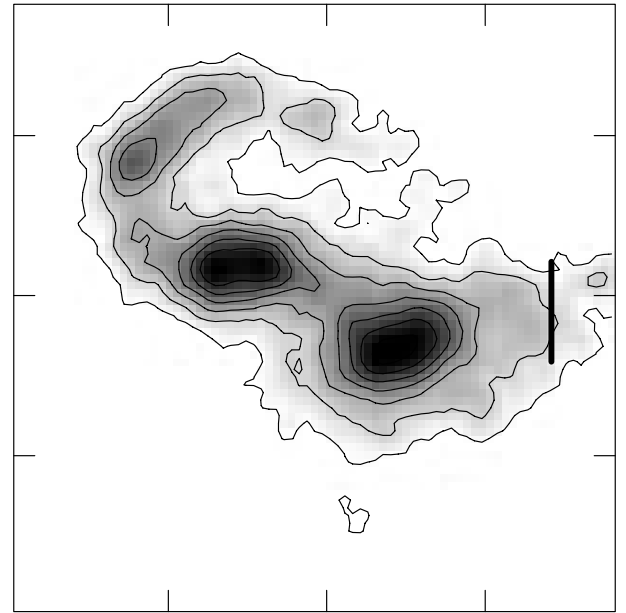

IRAS $06035-7102$

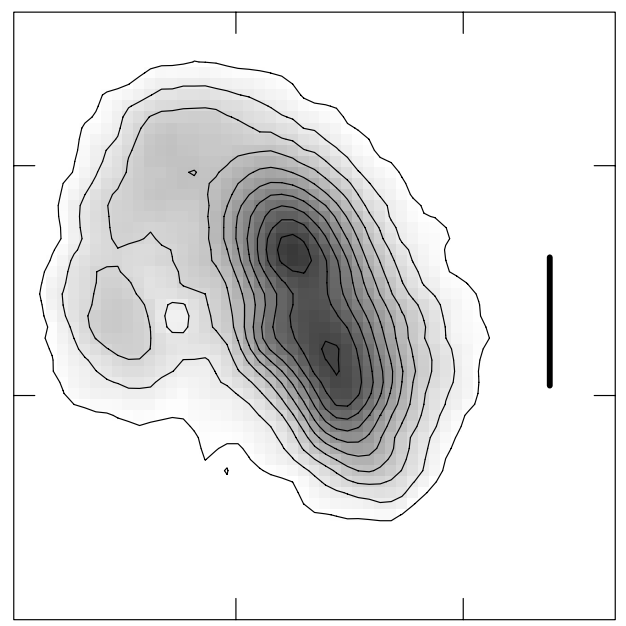

IRAS $06206-6315$

Fig. 3. $R$ band images of the Southern Ultraluminous Infrared Galaxies. To the left: greyscale image of the field around the system. The tickmarks are separated by $15^{\prime \prime}$. The vertical bar corresponds to $50 \mathrm{kpc}$. To the right: contour map of the central region. The tickmarks are separated by $5^{\prime \prime}$. The vertical bar corresponds to $5 \mathrm{kpc}$. In all images, North is up and East left 


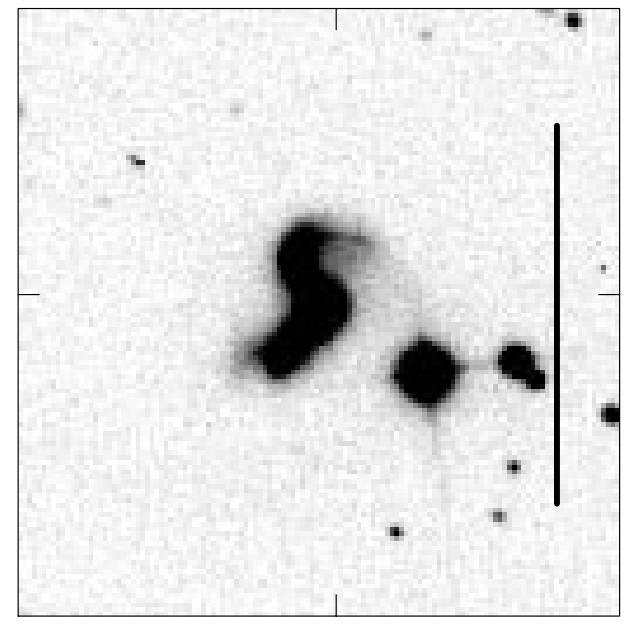

IRAS 09061-1248

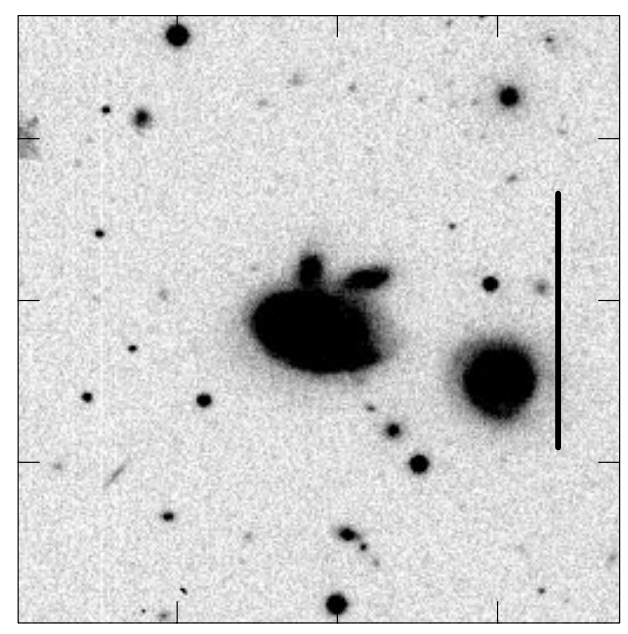

IRAS 09111-1007

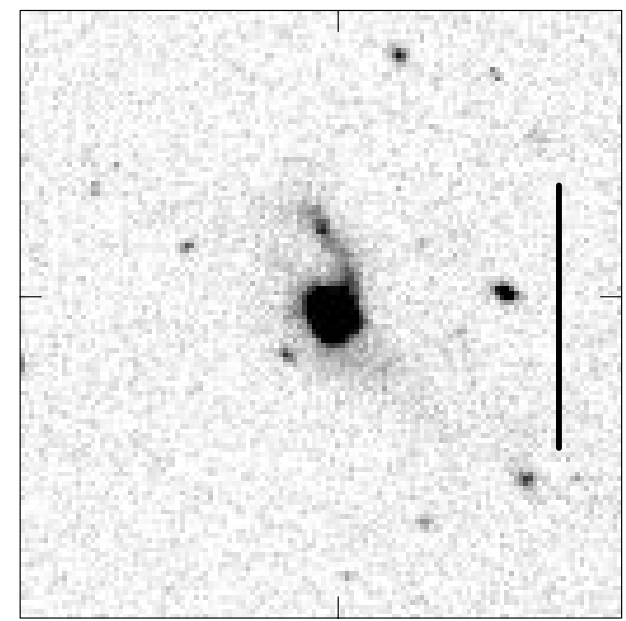

IRAS 11095-0238

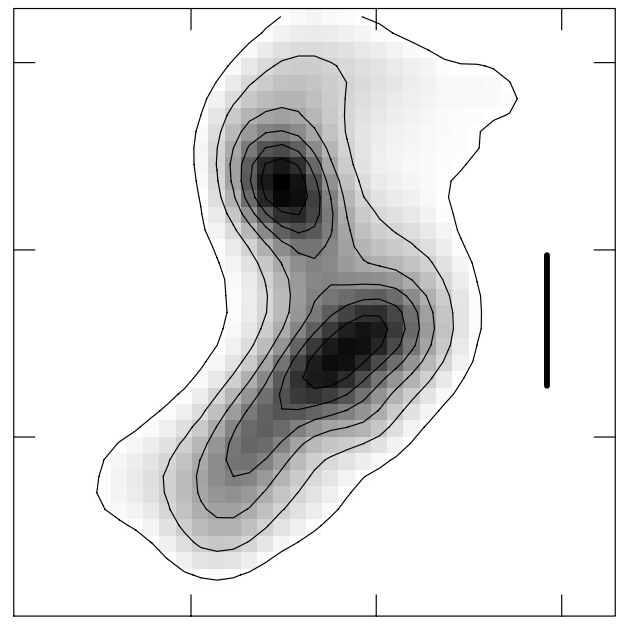

IRAS 09061-1248

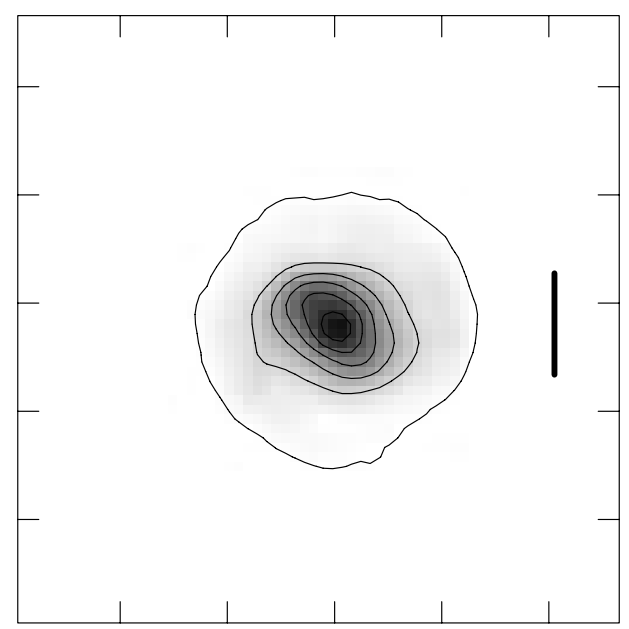

IRAS 09111-1007

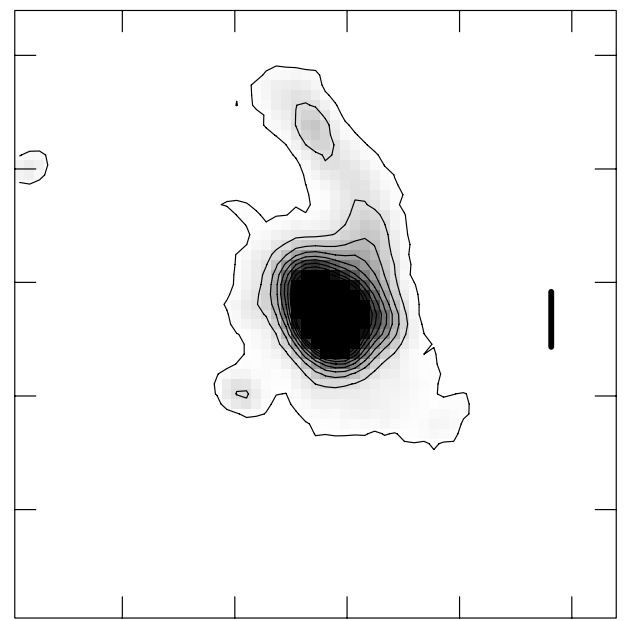

IRAS 11095-0238

Fig. 3. continued 


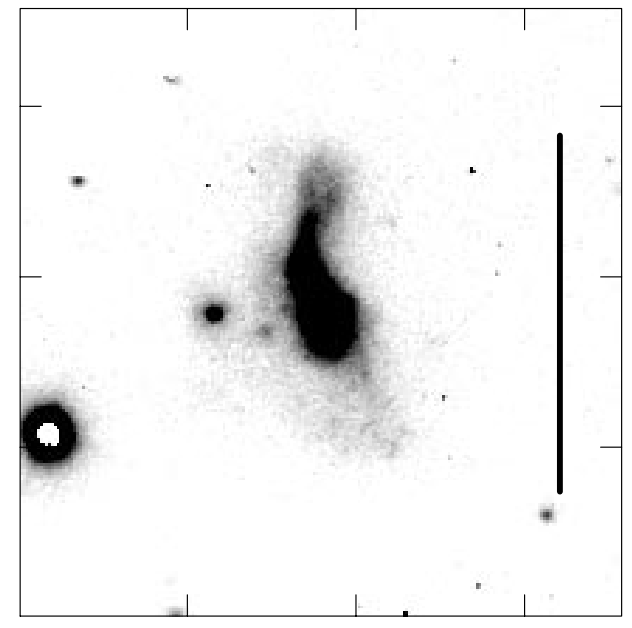

IRAS 14348-1447

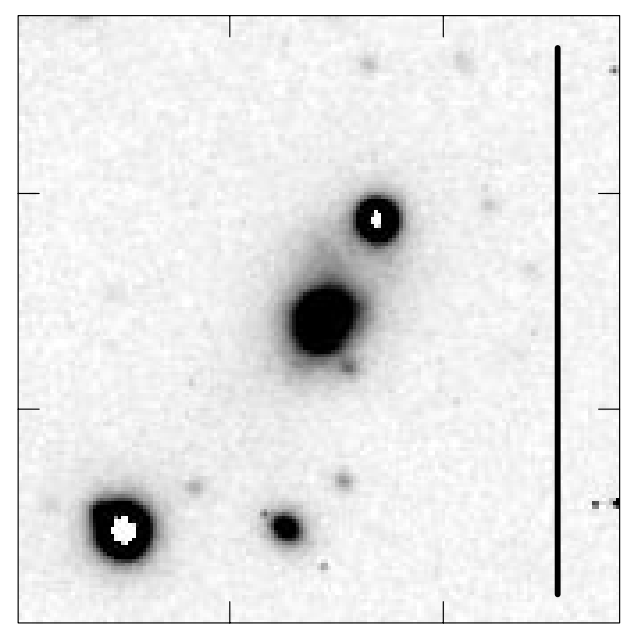

IRAS 14378-3651

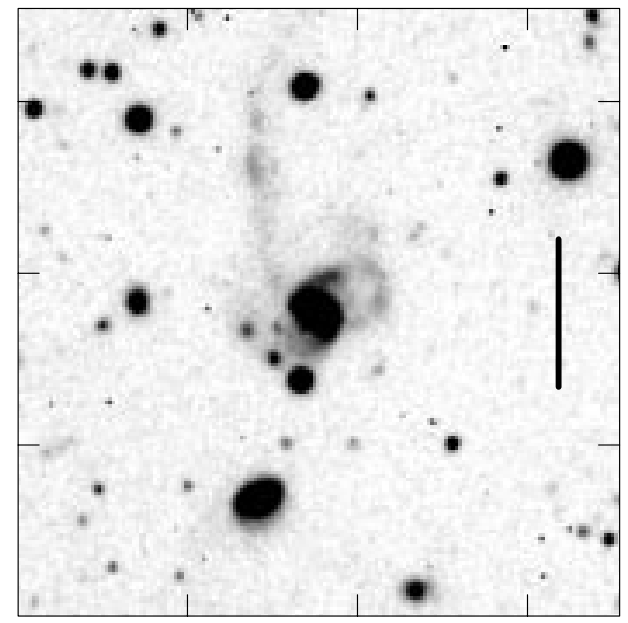

IRAS 15462-0450

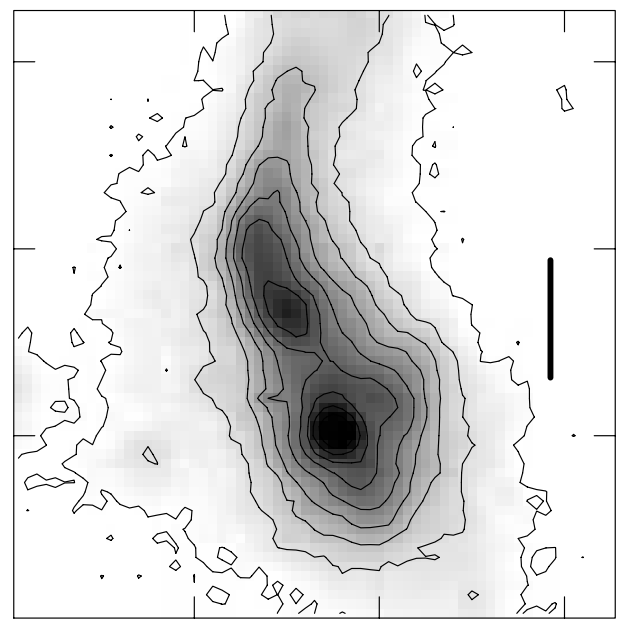

IRAS 14348-1447

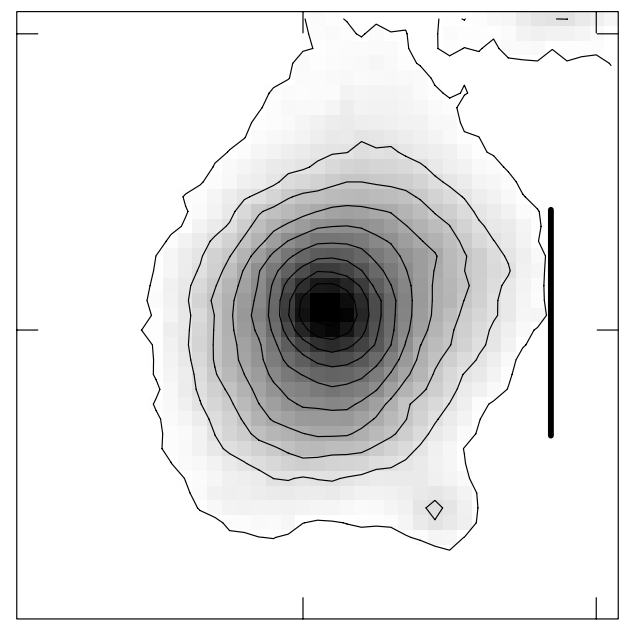

IRAS 14378-3651

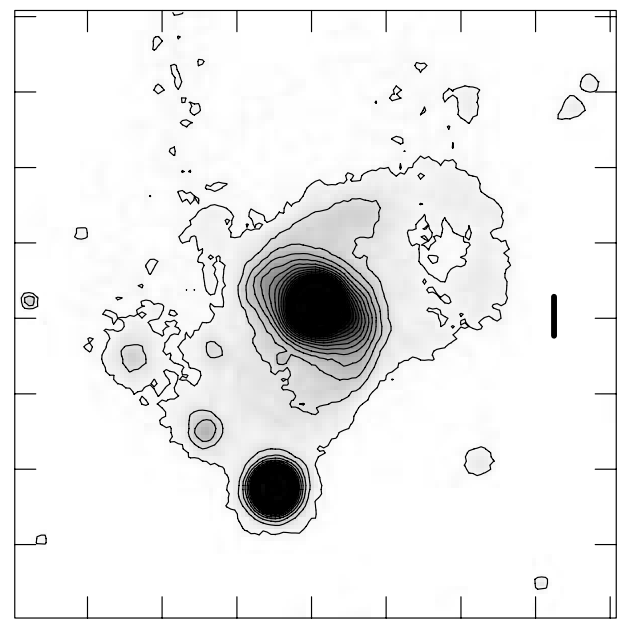

IRAS 15462-0450

Fig. 3. continued 


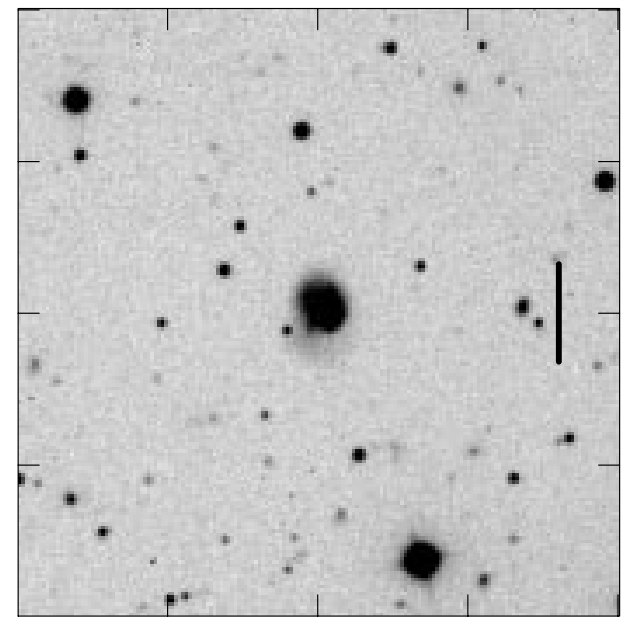

IRAS 16090-0139

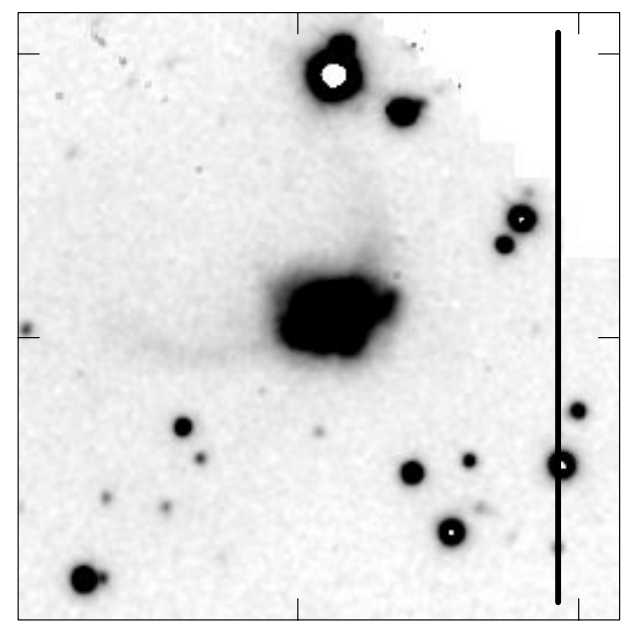

IRAS 17208-0014

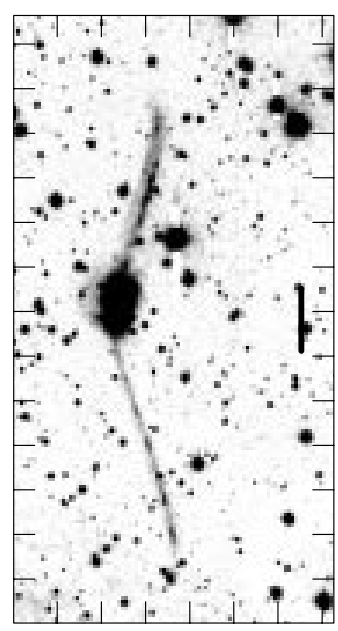

IRAS 19254-7245

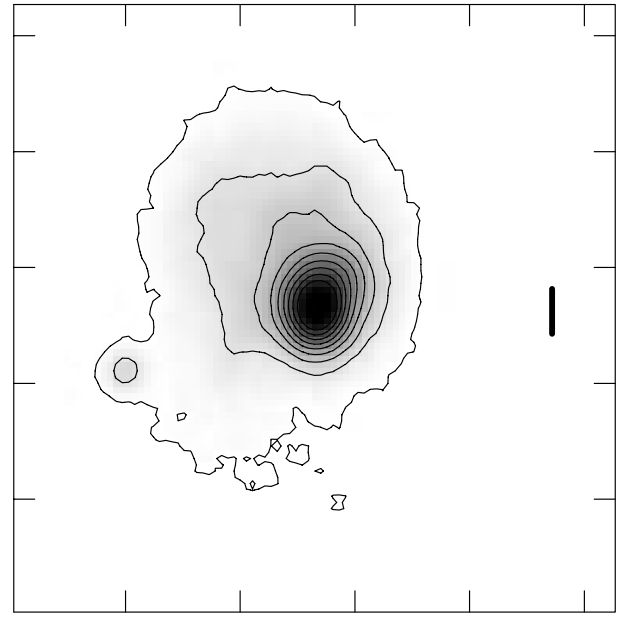

IRAS 16090-0139

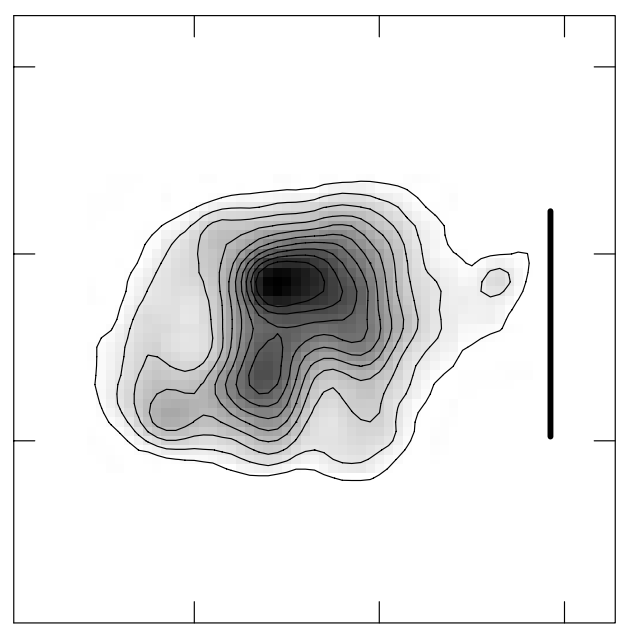

IRAS 17208-0014

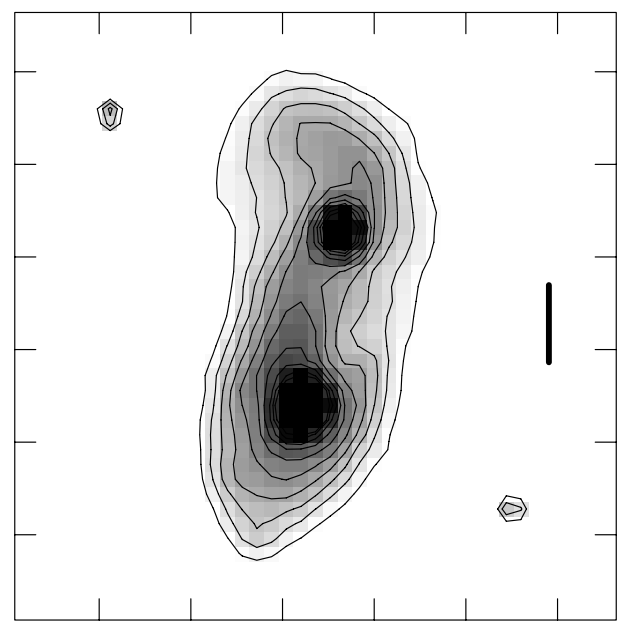

IRAS 19254-7245

Fig. 3. continued 


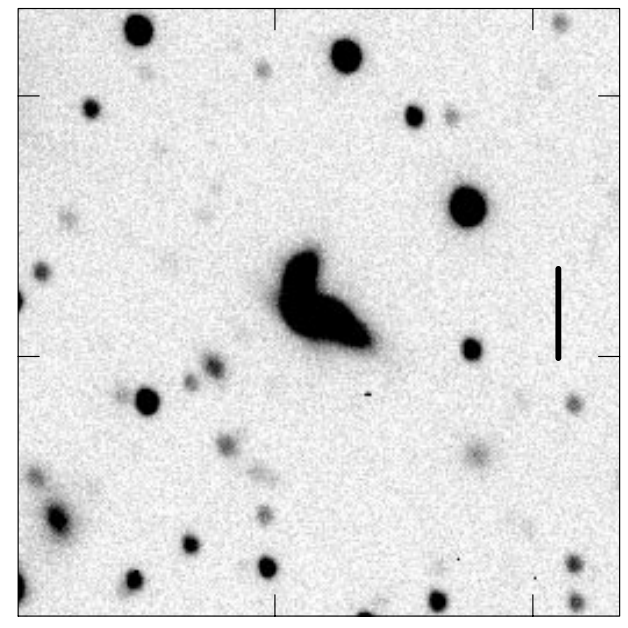

IRAS 20046-0623

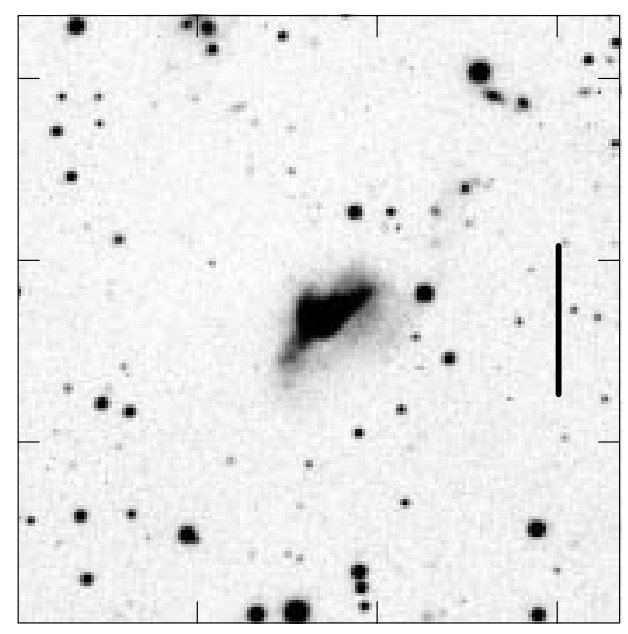

IRAS 20087-0308

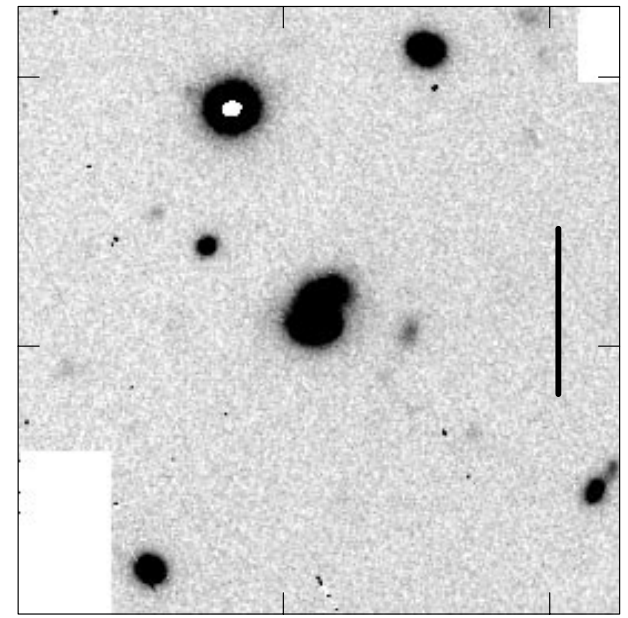

IRAS 20100-4156

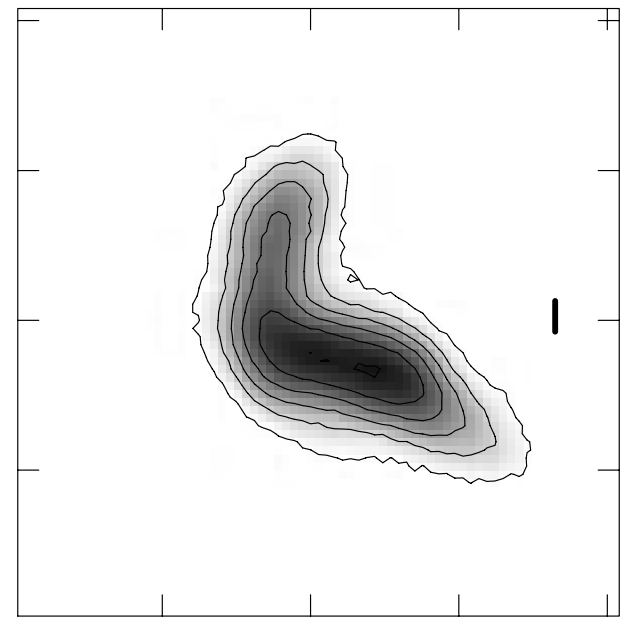

IRAS 20046-0623

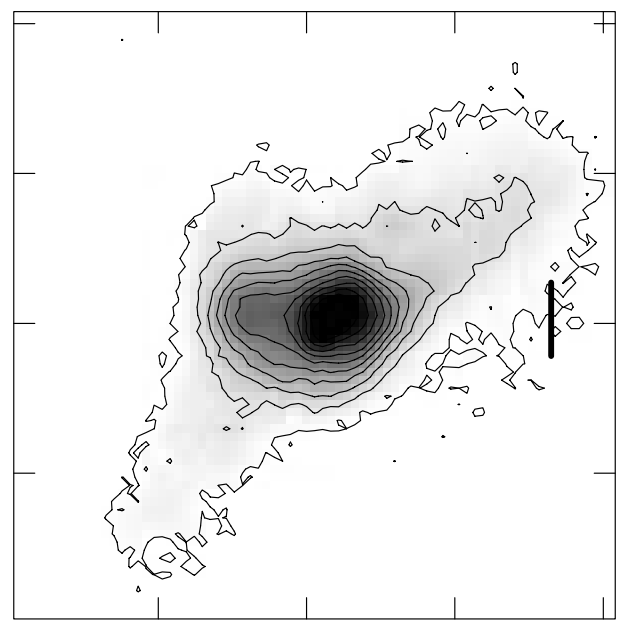

IRAS 20087-0308

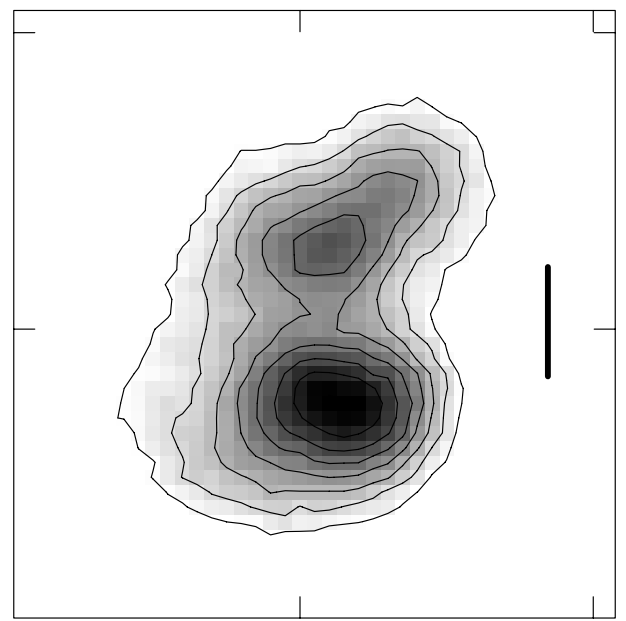

IRAS 20100-4156

Fig. 3. continued 


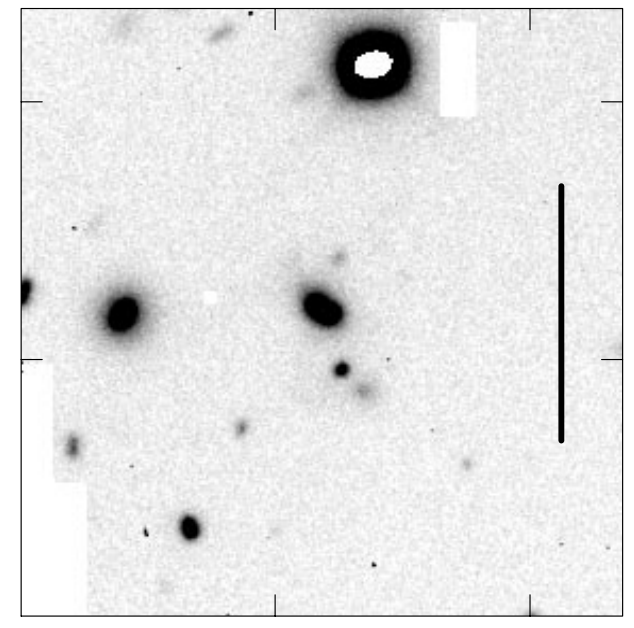

IRAS 20414-1651

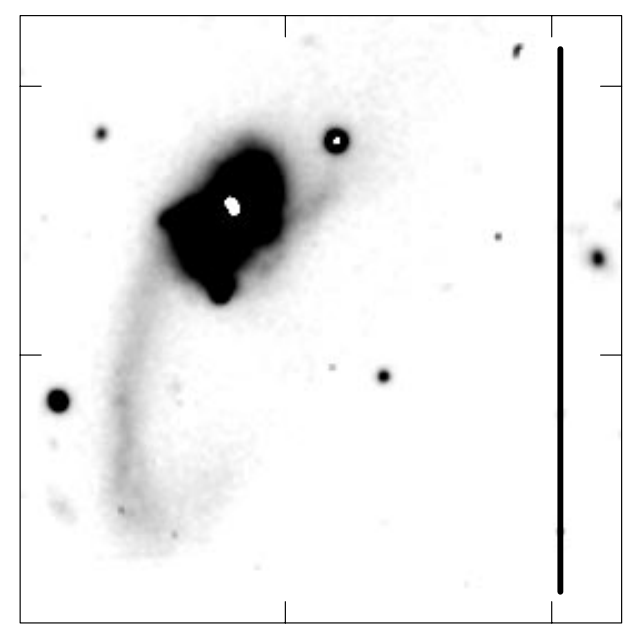

IRAS 20551-4251

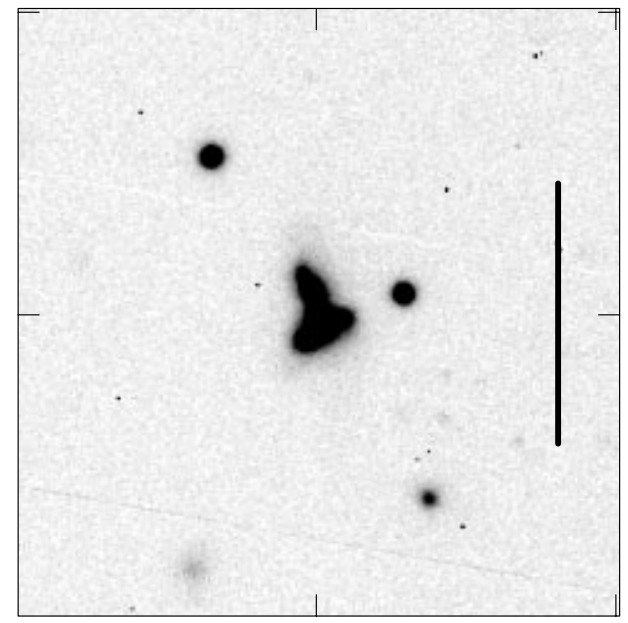

IRAS 21130-4446

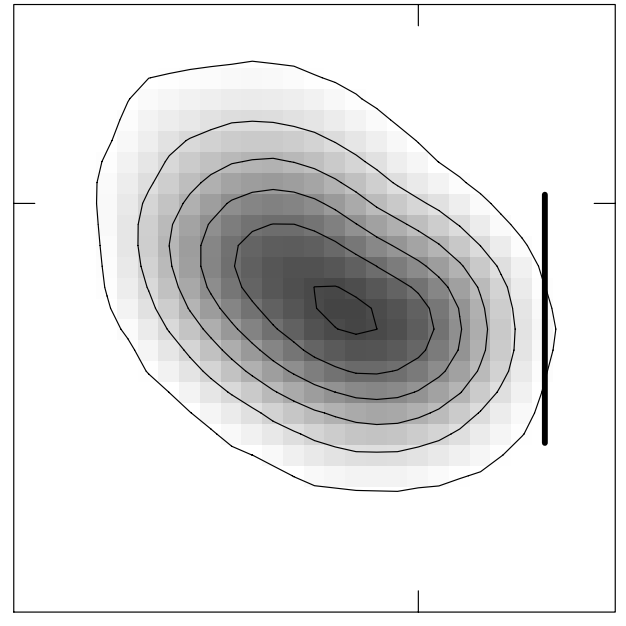

IRAS 20414-1651

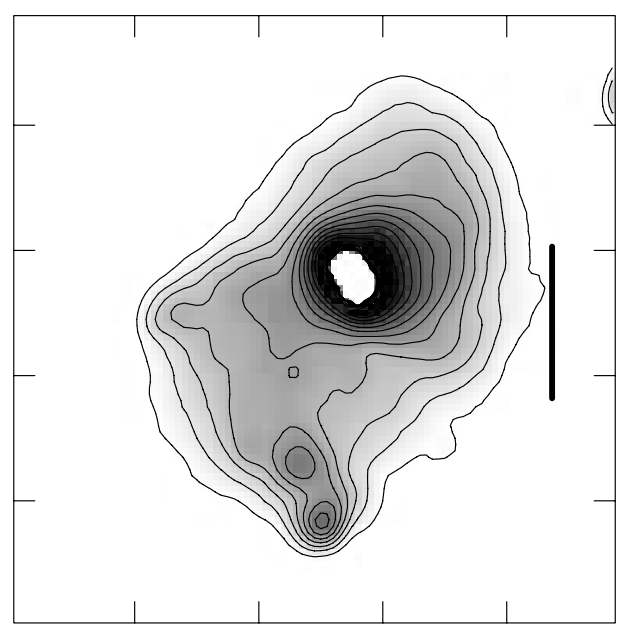

IRAS 20551-4251

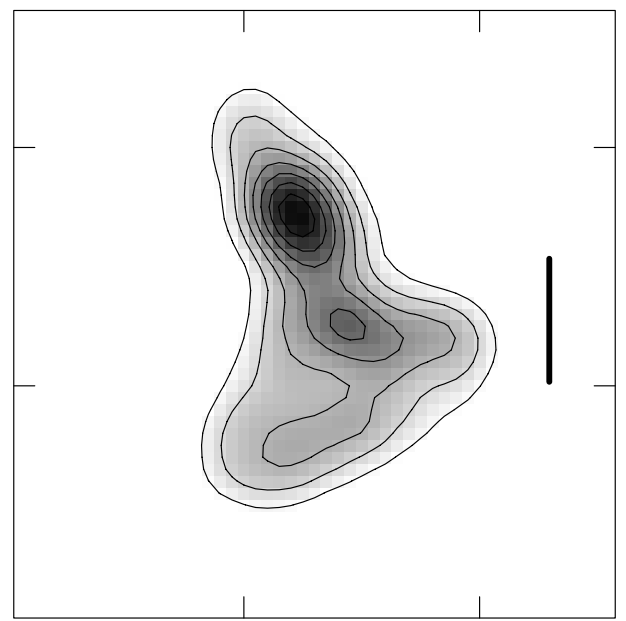

IRAS 21130-446

Fig. 3. continued 


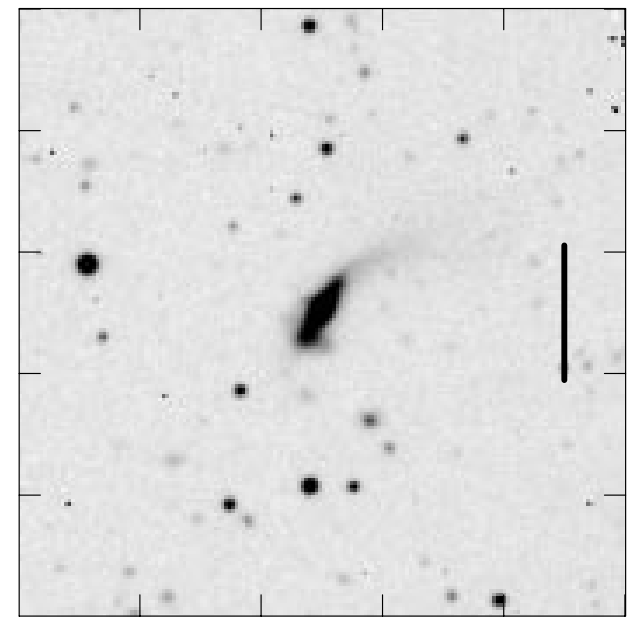

IRAS 21504-0628

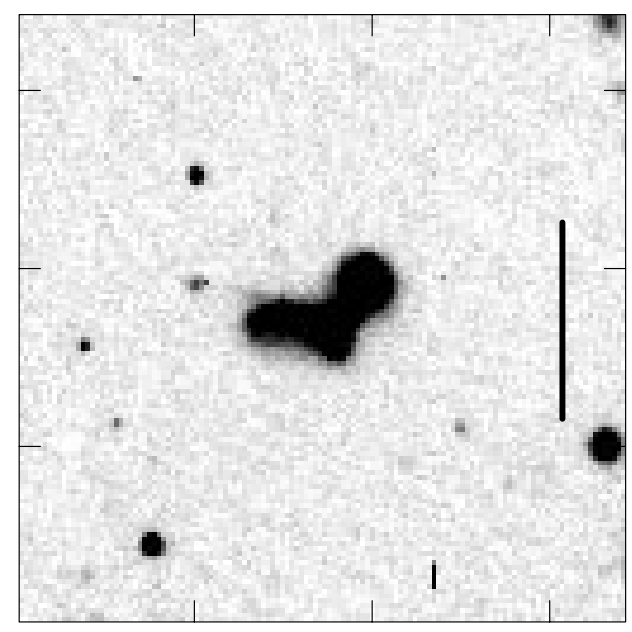

IRAS 22491-1808

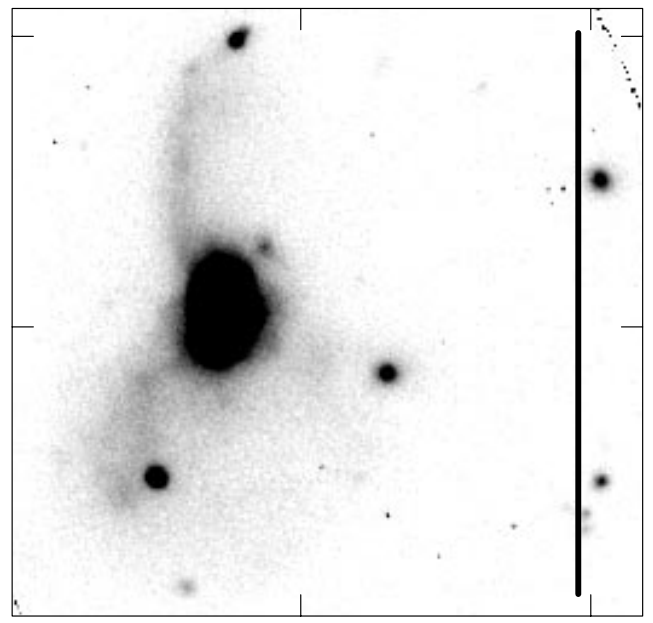

IRAS 23128-5919

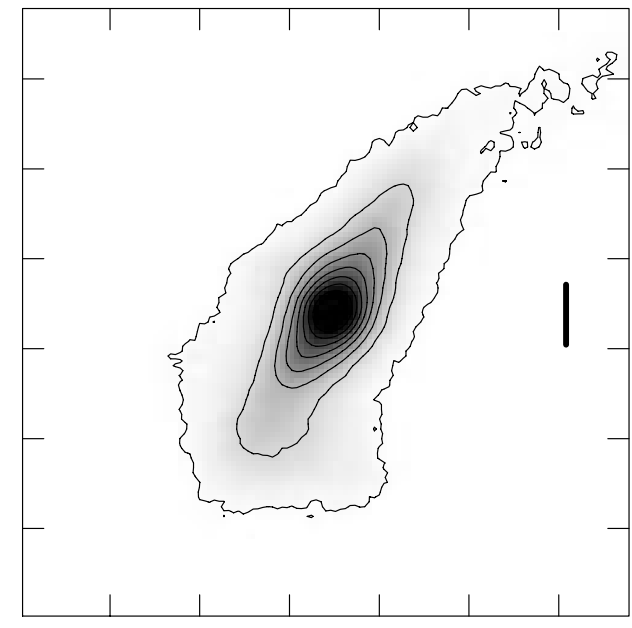

IRAS 21504-0628

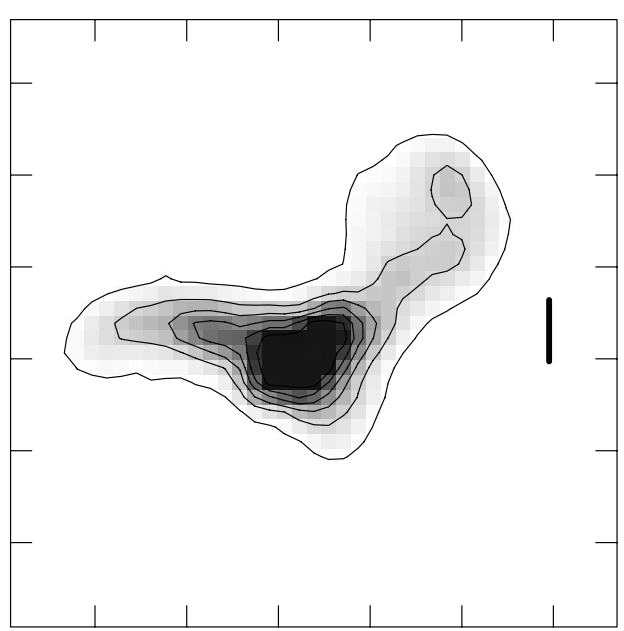

IRAS 22491-1808

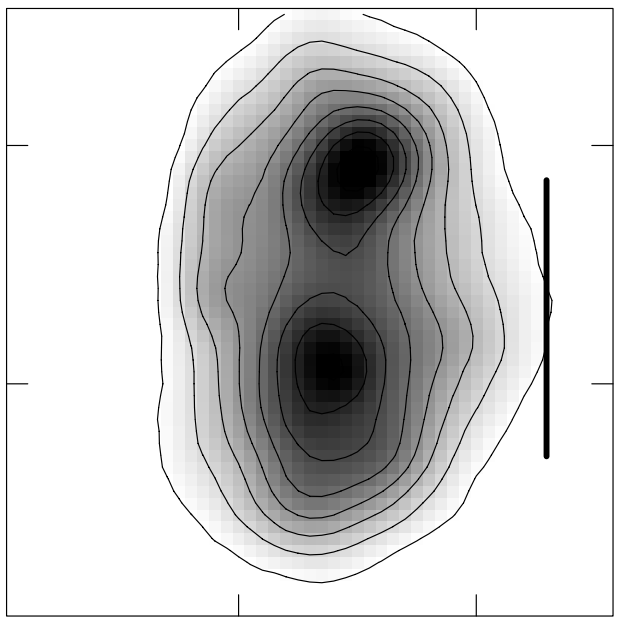

IRAS 23128-5919

Fig. 3. continued 


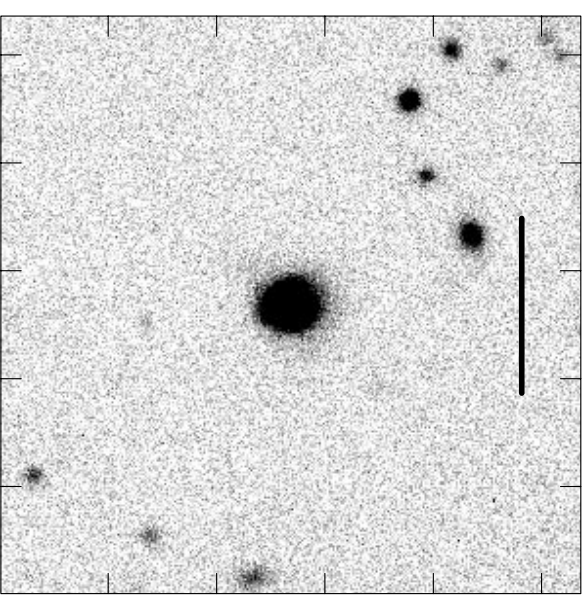

IRAS 23230-6926

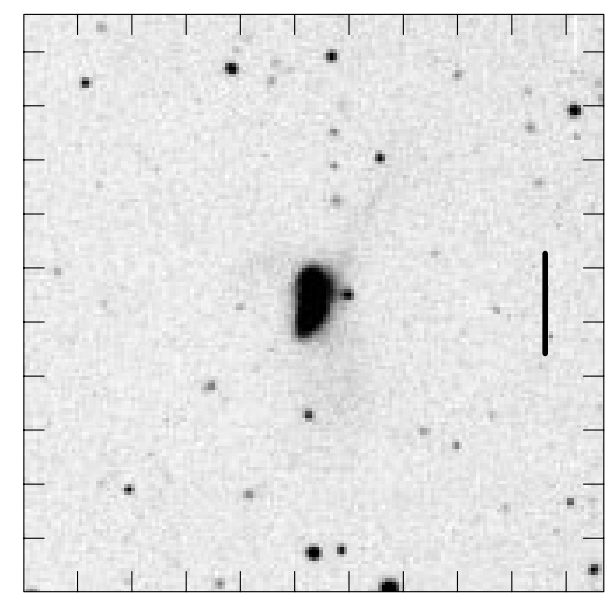

IRAS 23389-6139

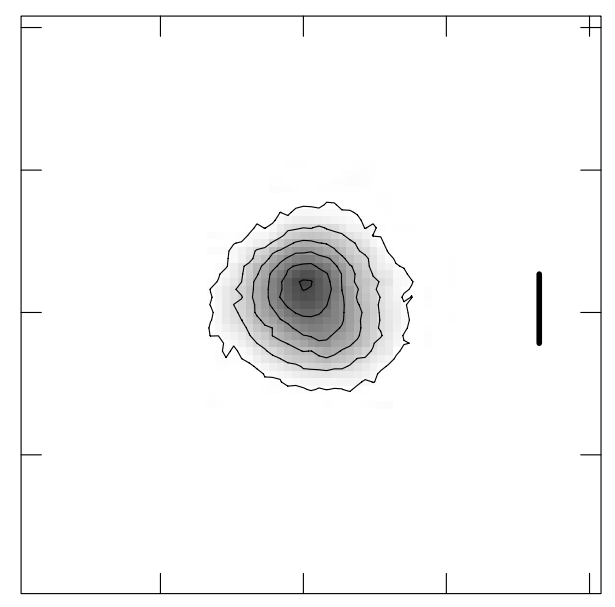

IRAS 23230-6926

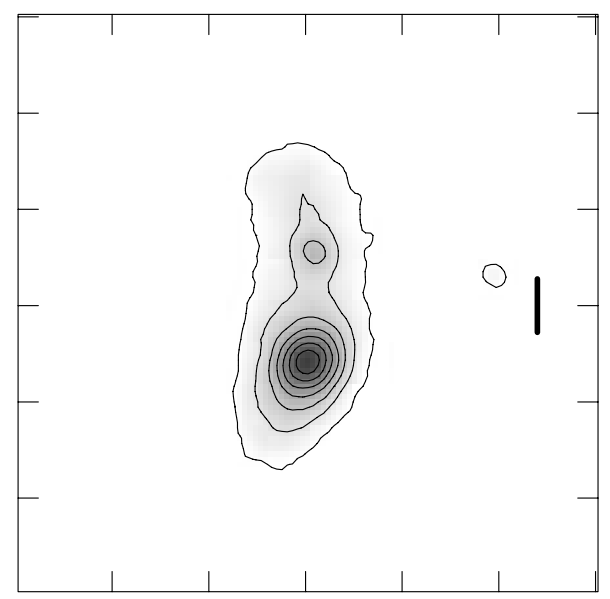

IRAS 23389-6139

Fig. 3. continued 


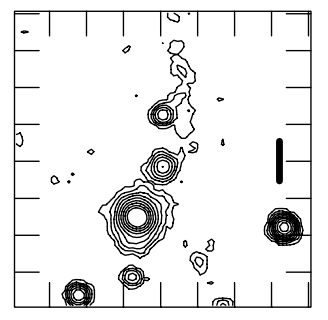

IRAS 00199-7426

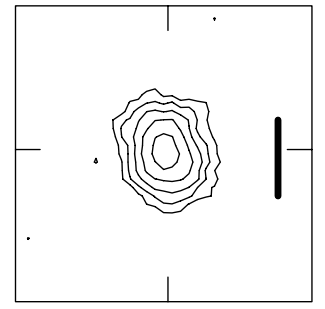

IRAS 11095-0238

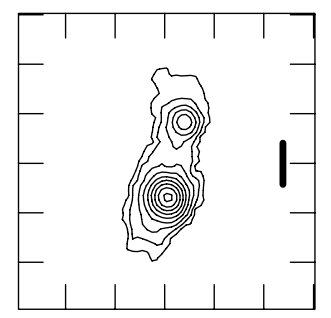

IRAS 19254-7245

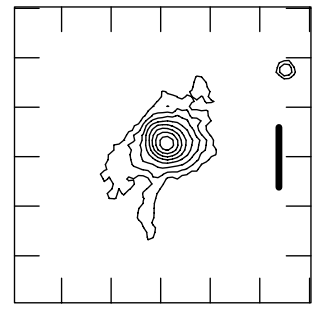

IRAS 20551-4251

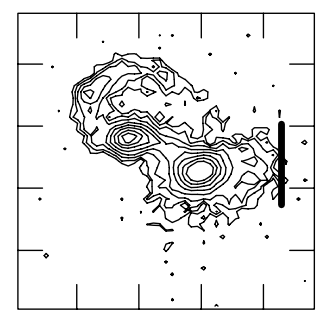

IRAS 06035-7102

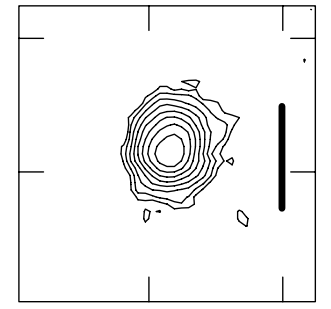

IRAS 14378-3651

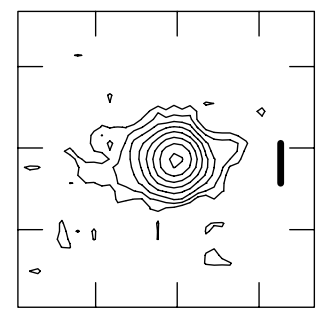

IRAS 20087-0308

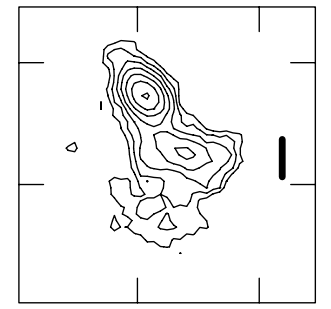

IRAS 21130-4446

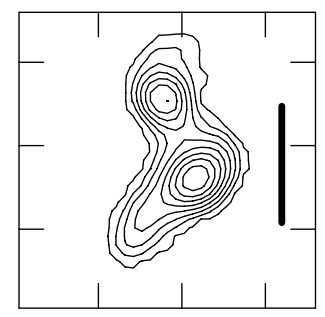

IRAS 09061-1248

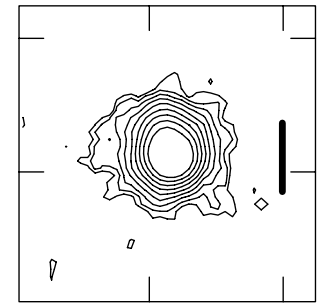

IRAS 15462-0450

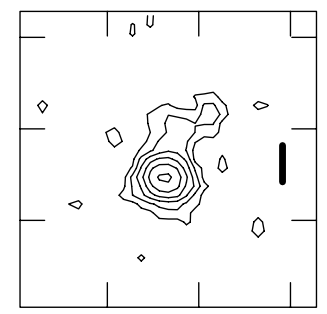

IRAS 20100-4156

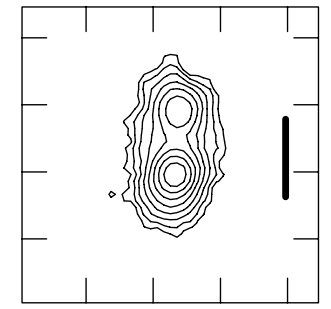

IRAS 23128-5919

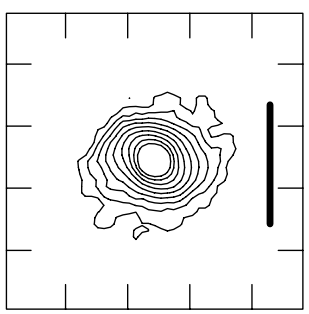

IRAS 09111-1007

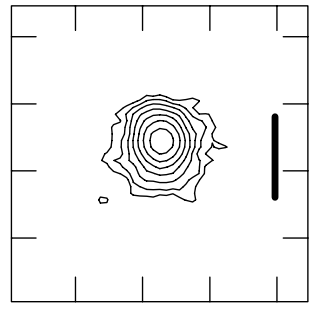

IRAS 17208-0014

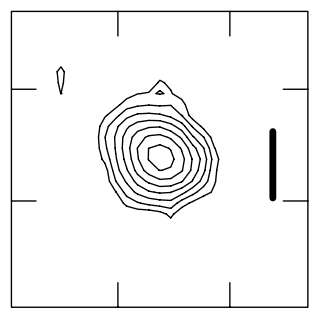

IRAS 20414-1651

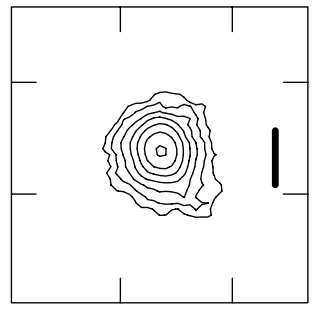

IRAS 23230-6926

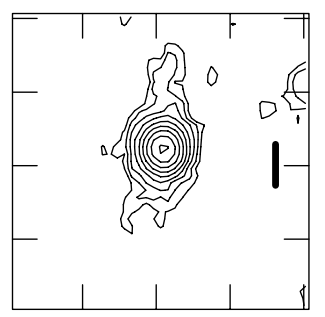

IRAS 23389-6139

Fig. 4. Calibrated $K$ band contour maps of the Southern Ultraluminous Infrared Galaxies. North is up and East left. The tickmarks are separated by $5^{\prime \prime}$. The vertical bar corresponds to $5 \mathrm{kpc}$. The lower contour is $18.5 \mathrm{mag} / \square^{\prime \prime}$. The step is $0.5 \mathrm{mag} / \square^{\prime \prime}$ 

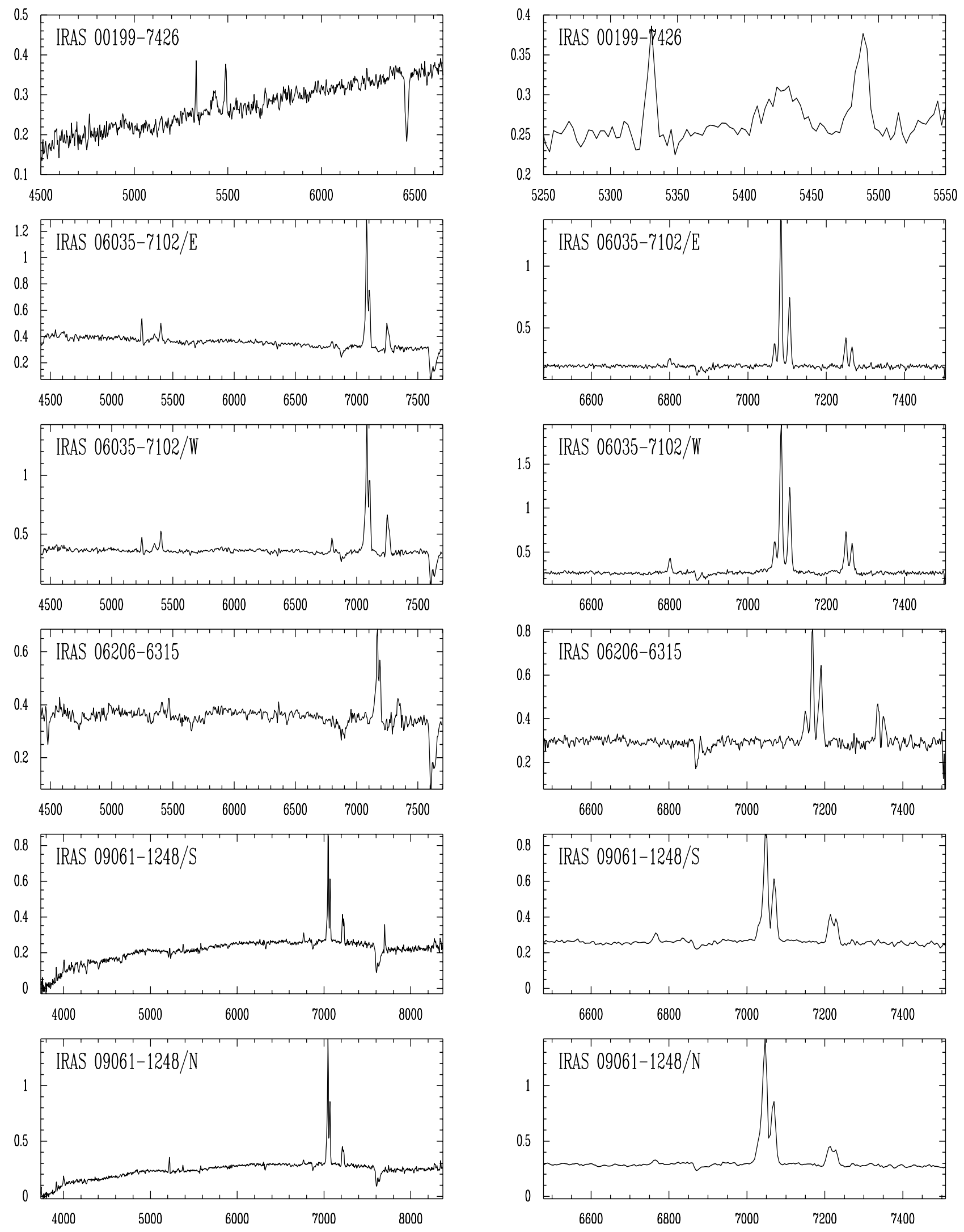

Fig. 5. Optical spectra of the Southern Ultraluminous Infrared Galaxies. To the left: low resolution spectra, to the right: high resolution red spectra. For IRAS 00199 - 7426, IRAS 09061 - 1248, IRAS 09111 - 1007 and IRAS 15462 - 0450 , a close up of the LR spectrum in the blue, near the $\mathrm{H}_{\beta}$ line or in the red, near the $\mathrm{H}_{\alpha}$ line, is presented instead of the HR spectrum. The units are $10^{-15} \mathrm{erg} \mathrm{cm}^{-2} \mathrm{~s}^{-1} \AA^{-1}$ 

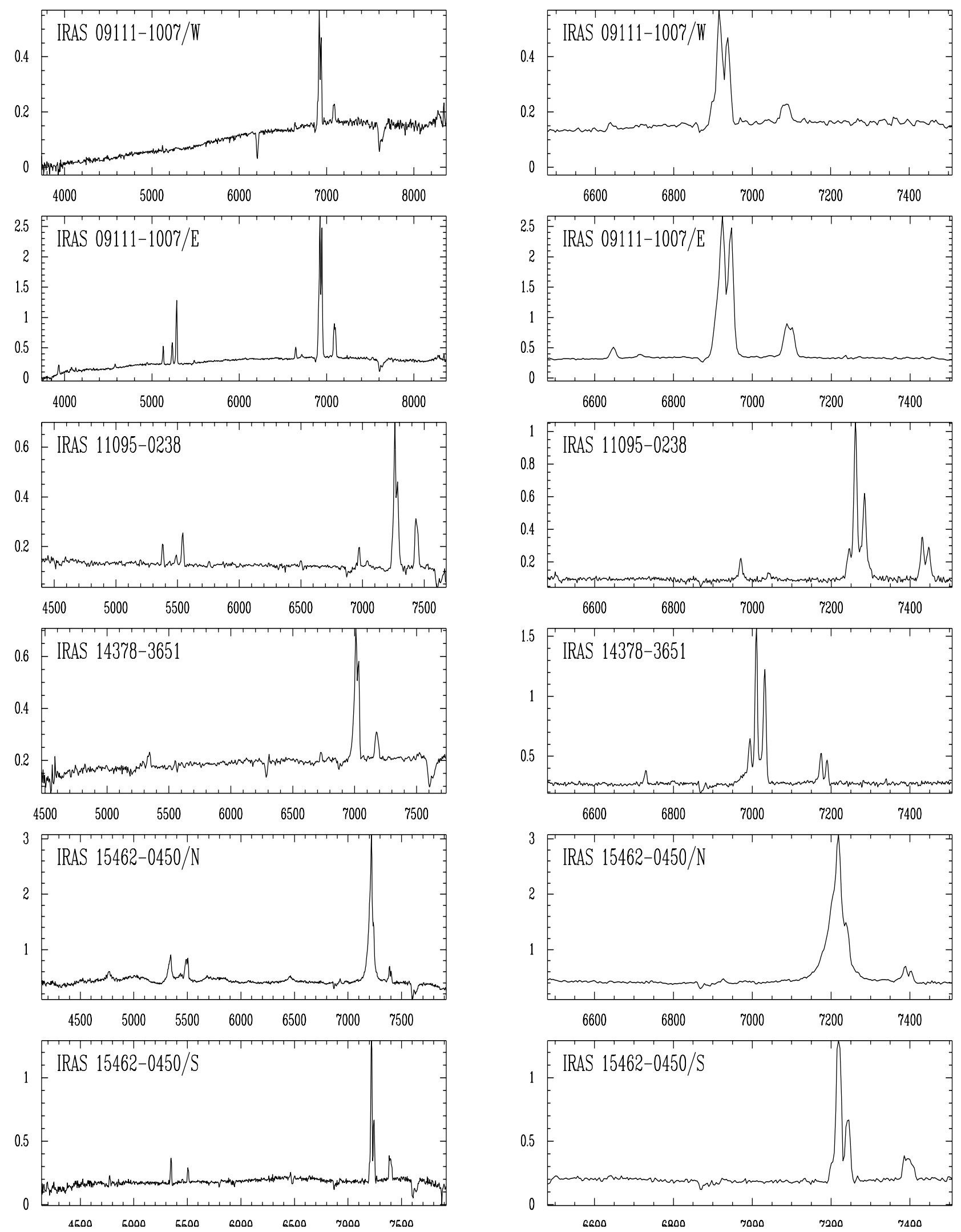

Fig. 5. continued 

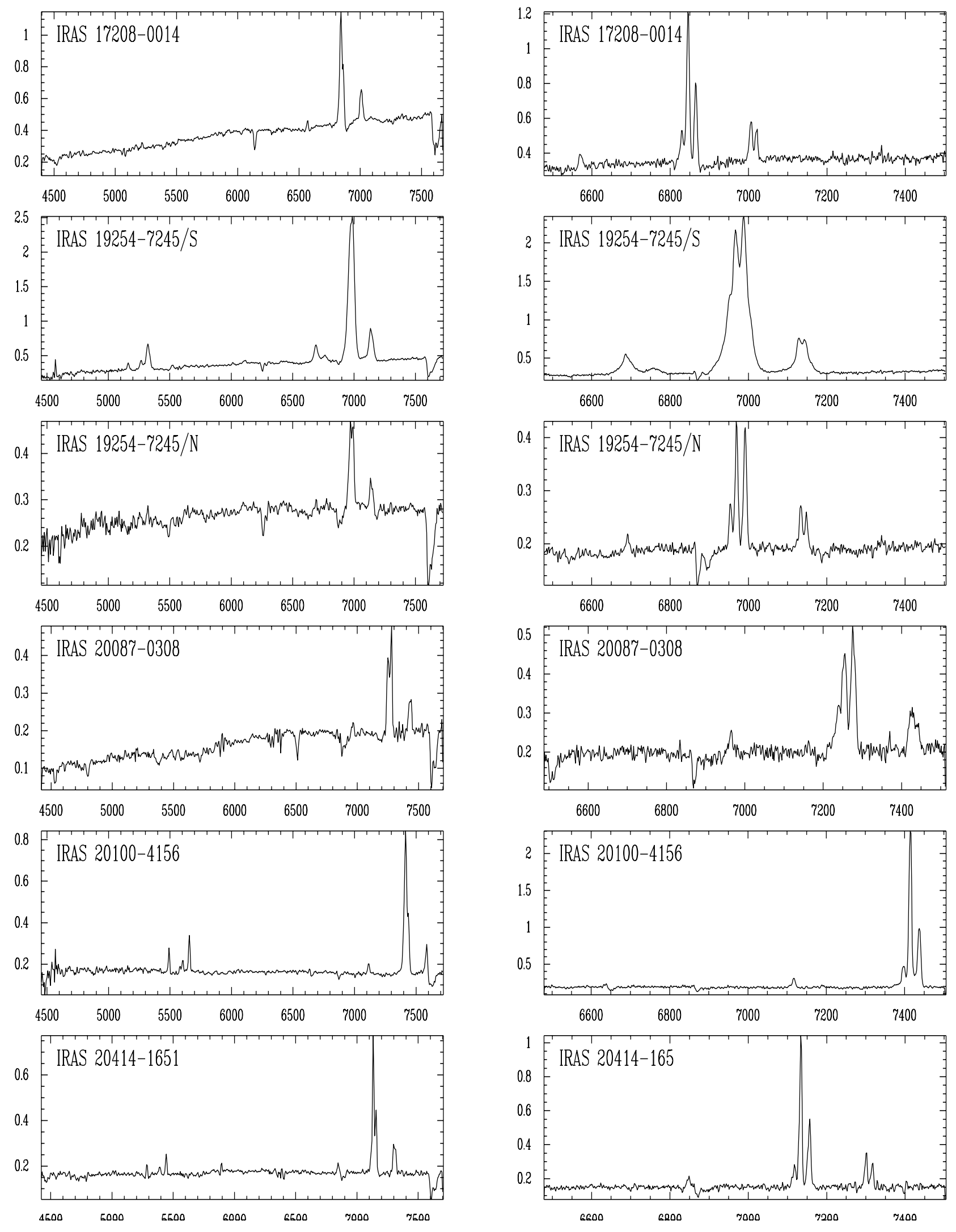

Fig. 5. continued 

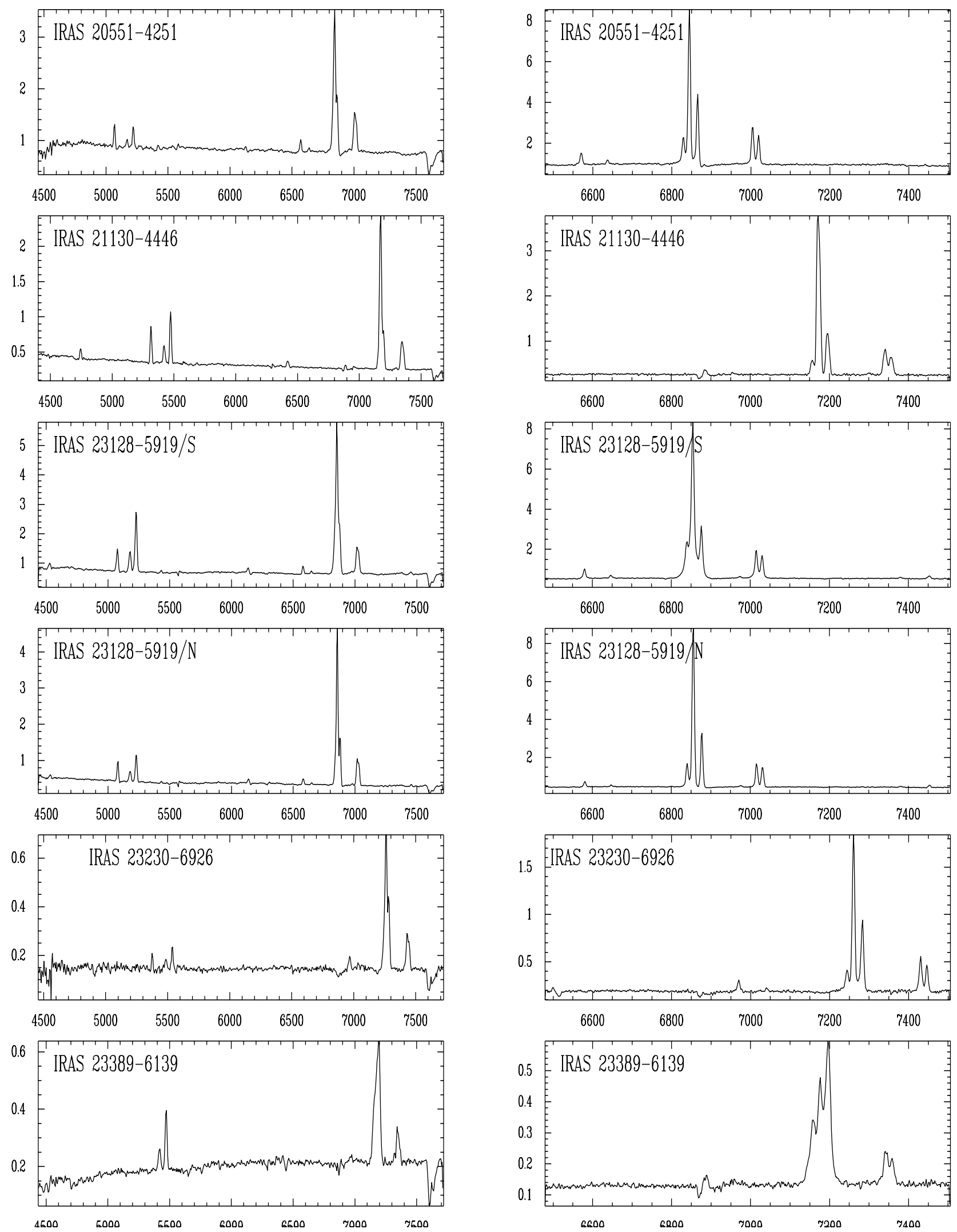

Fig. 5. continued 


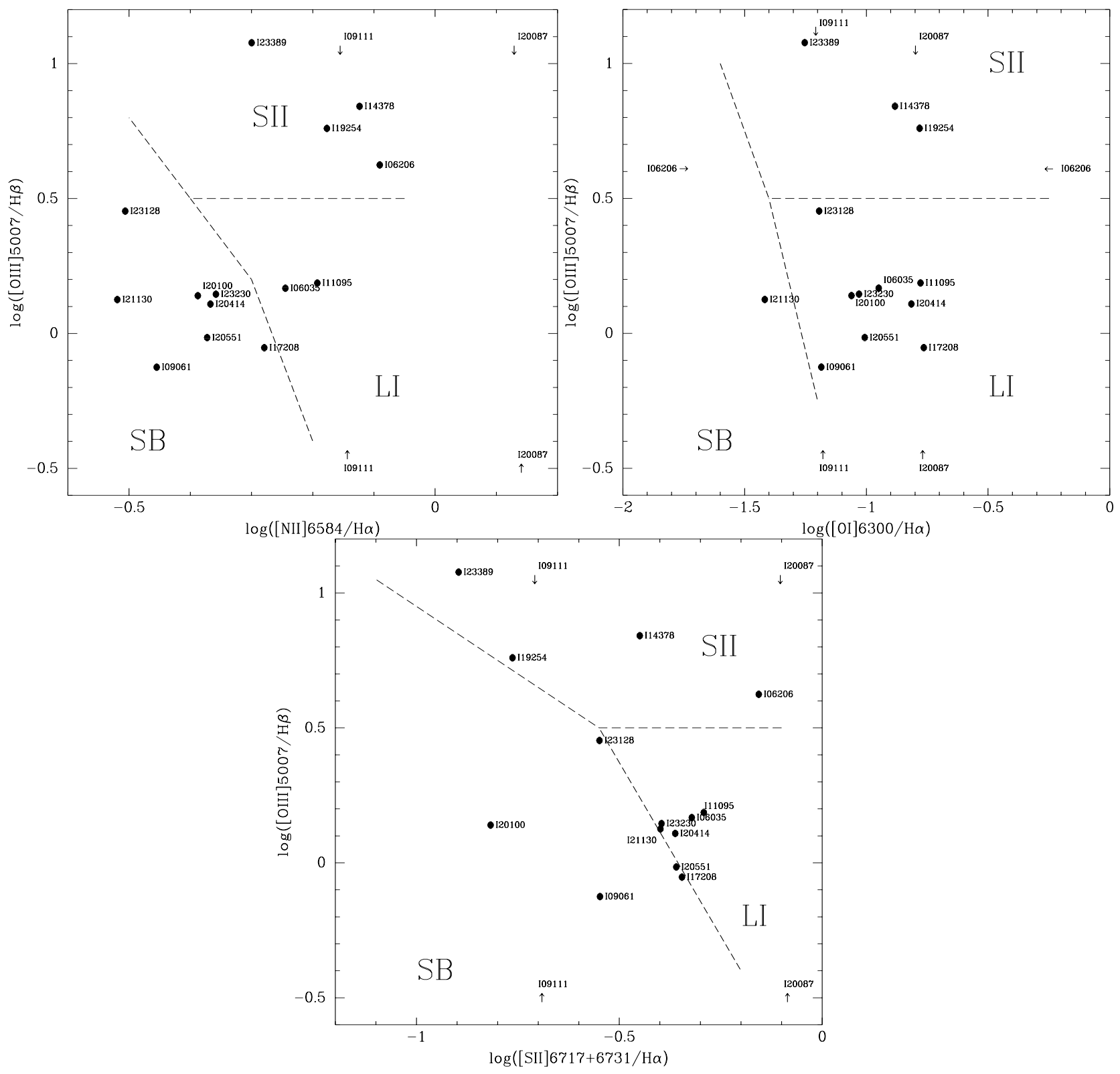

Fig. 6. Diagnostic diagrams for spectral classification (Veilleux \& Osterbrock 1987). The line ratios have been corrected for galactic and interstellar absorption 